\title{
On Normal Bases of Some Ring Extensions in Number Fields I
}

\author{
Fuminori KAWAMOTO
}

Gakushuin University

\section{Introduction.}

Let $k$ be a number field and $K / k$ a finite Galois extension with Galois group $G=\operatorname{Gal}(K / k)$. For a number field $N, \mathfrak{o}_{N}$ denotes the ring of integers in $N$. Let $S$ be a finite set of prime ideals of $\mathfrak{o}_{k}$ that contains all prime ideals which are wildly ramified in $K / k$. For a finite extension $N / k$, we simply denote by $\mathfrak{o}_{N}(S)$ the ring of elements $a$ in $N$ with $\operatorname{ord}_{\mathfrak{P}}(a) \geq 0$ for all prime ideals $\mathfrak{P}$ of $\mathfrak{o}_{N}$, not lying above $S$. The field $K$ can be regarded as a module over the group ring $k G$ of $G$ over $k$ by the action $\alpha^{\lambda}=\sum_{s \in G} a_{s} \alpha^{s}$ for $\alpha \in K$ and $\lambda=\sum_{s \in G} a_{s} s \in k G$. We say that a ring extension $\mathfrak{o}_{K}(S) / \mathfrak{o}_{k}(S)$ has a normal basis if $\mathfrak{o}_{K}(S)$ is a free $\mathfrak{o}_{k}(S)$ [G]-module, that is, there exists some $\alpha$ in $\mathfrak{o}_{K}(S)$ such that $\left\{\alpha^{s}\right\}_{s \in G}$ is a free $\mathfrak{o}_{k}(S)$-basis of $\mathfrak{o}_{K}(S)$. The extension $\mathfrak{o}_{K}(S) / \mathfrak{o}_{k}(S)$ is called ramified if there exists some prime ideal of $\mathfrak{o}_{k}$, not belonging to $S$, which is ramified in $K / k$ (this means that such prime ideal of $\mathfrak{o}_{k}$ is ramified in the Dedekind ring extension $\mathfrak{o}_{K} / \mathfrak{o}_{k}$, as usual). If not so, then it is called unramified.

We remark the following fact on the existence of normal bases of extensions of the rings of $S$-integers which was pointed out by $\mathbf{H}$. Suzuki and whose proof is due to him. It says that we can take a sufficiently large set $U \cup S$, keeping the ramification of primes outside $S$, such that $\mathfrak{o}_{K}(U \cup S) / \mathfrak{o}_{k}(U \cup S)$ has a normal basis.

Proposition 1.1. Let the notations be as above and $T(\neq \varnothing)$ a finite set of prime ideals of $\mathfrak{o}_{k}$ that contains all prime ideals, not belonging to $S$, which are ramified in $K / k$. Then there exists a finite set $U$ of prime ideals of $\mathfrak{o}_{k}$ such that $U \cap T=\varnothing$ and $\mathfrak{o}_{K}(U \cup S) /$ $\mathfrak{o}_{k}(U \cup S)$ has a normal basis.

Proof. Let $V:=\mathfrak{o}_{k}-\bigcup_{\mathfrak{p} \in T} \mathfrak{p}$ be a multiplicative subset of $\mathfrak{o}_{k}$ and $V^{-1} \mathfrak{o}_{k}$ a ring of quotients of $\mathfrak{b}_{k}$. Then $V^{-1} \mathfrak{v}_{k}$ is a semi-local ring with maximal ideals $\left\{\mathfrak{p} \cdot\left(V^{-1} \mathfrak{o}_{k}\right)\right\}_{\mathfrak{p} \in T}$ and $V^{-1} \mathfrak{o}_{K}$ is a $\left(V^{-1} \mathfrak{o}_{k}\right)[G]$-module. Since all primes in $T$ are tamely ramified, there exists some $\alpha$ in $\mathfrak{o}_{K}$ such that $1 \otimes \alpha$ is a free generator of $\mathfrak{o}_{k_{\mathrm{p}}} \otimes_{\mathfrak{o}_{k}} \mathfrak{o}_{k}$ over $\mathfrak{o}_{\boldsymbol{k}_{\mathrm{p}}} G$ for each $\mathfrak{p} \in T$ (Cf. [8, Lemma 2.6]), where $\mathfrak{o}_{k_{\mathfrak{p}}}$ denotes the valuation ring of the completion of 
$k$ with respect to $\mathfrak{p}$. Therefore $\alpha$ is also a free generator of $V^{-1} \mathfrak{o}_{K} \operatorname{over}\left(V^{-1} \mathfrak{o}_{k}\right)[G]$. Put $M:=\mathfrak{o}_{K} /\left(\mathfrak{o}_{k} G \cdot \alpha\right)$. Then $M$ has a finite number of generators over $\mathfrak{o}_{k}$, say $m_{1}, \cdots, m_{r}$. Since $V^{-1} M=0$, there is some $u_{i}$ in $V$ for each $i$ such that $u_{i} m_{i}=0$. If we put $u=$ $\prod_{i=1}^{r} u_{i}$, then we have $\langle u\rangle^{-1} M=0$ where $\langle u\rangle$ denotes a multiplicative subset of $\mathfrak{o}_{k}$, generated by $u$. Let $U$ be a set of prime divisors of $u$. Then $U \cap T=\varnothing$ and $\mathfrak{o}_{k}(U) \bigotimes_{\mathrm{o}_{k}} M=$ $\mathfrak{o}_{k}(U) \otimes_{\langle u\rangle^{-1} \mathfrak{o}_{k}}\langle u\rangle^{-1} M=0$. So $\mathfrak{o}_{K}(U)=\mathfrak{v}_{k}(U)[G] \cdot \alpha$. This proves our proposition.

From now on, assume that $K / k$ is abelian and let $\hat{G}$ be the group of characters of $G$. In the previous paper [8], for each $\chi \in \hat{G}$, an ideal $\mathfrak{b}(\chi)$ was defined by resolvents of elements of $K$ (for its definition, see Section 2) and we gave a necessary and sufficient condition for $\mathfrak{o}_{K}(S) / \mathfrak{o}_{k}(S)$ to have a normal basis in terms of these ideals. Since resolvents are connected with Gauss sums, Stickelberger's theorem gives an information on ideals $\mathfrak{b}(\chi)$. For this, we study a property of $\mathfrak{b}(\chi)$ in Section 2 . After Section 3 , we assume that $k$ is a totally real number field or a $C M$-field, i.e., a totally imaginary quadratic extension of a totally real number field. In comparison with Proposition 1.1, we can give also sequences $\left\{S_{n}\right\}$ of finite sets of prime ideals of $\mathfrak{o}_{k}$ with $S_{n} \subsetneq S_{n+1}$, such that $\mathfrak{o}_{K}\left(S_{n}\right) / \mathfrak{o}_{k}\left(S_{n}\right)$ does not have a normal basis for each positive integer $n$ (Propositions 4.3 and 4.5). This fact follows from results of Section 3 (Proposition 3.3 and Lemma 3.5) and a sufficient condition for the non-existence of normal basis of ramified ring extension $\mathfrak{o}_{K}(S) / \mathfrak{o}_{k}(S)$ which is given in Section 4 (Theorem 4.1). In Section 5 , let $K$ be an abelian field with prime conductor over the field $\mathbf{Q}$ of rational numbers. Then using Proposition 4.3, we discuss a normal basis of $\mathfrak{o}_{K} / \mathfrak{o}_{k}(S=\varnothing)$ (Theorem 5.3). When $K$ is the $p$ th cyclotomic field, $p$ being an odd prime, and $[K: k]$ is a prime, a normal basis of $\mathfrak{o}_{K} / \mathfrak{o}_{k}$ was studied by Cougnard [4, 5] and Brinkhuis [2]. Theorem 5.3 generalizes their result. It should be noted that our results in Section 4, 5 are a development of Brinkhuis' idea [2].

Throughout this paper, the above and following notations are used. For a number field $N$ and each $\chi \in \hat{G}, N(\chi)$ denotes the field generated by the values of $\chi$ on $G$ over $N$. For a ring $R$ and a group $\Gamma$, we denote by $R \Gamma$ (or $R[\Gamma]$ ) the group ring of $\Gamma$ over $R$ and by $R^{\times}$the group of units in $R$. For a set $R,|R|$ denotes the cardinal of $R$. For a positive integer $n, \zeta_{n}$ denotes a primitive $n$th root of unity. We denote by $\mathbf{Z}$ and $\mathbf{R}$ the ring of rational integers and the field of real numbers, respectively. For a number field $N$, we denote by $N^{+}$the maximal real subfield of $N: N^{+}:=N \cap \mathbf{R}$. For an integral divisor $\mathrm{n}$ of $k, k(\mathfrak{n})$ denotes the ray class field of $k \bmod n$. Specially $\tilde{k}:=k(1)$ is the Hilbert class field of $k$. Let $n_{0}$ and $n_{\infty}$ denote the finite and infinite components of $n$, respectively.

ACKNOWLEDGments. The author would like to thank Dr. Suzuki for pointing out Proposition 1.1 and a certain person for his/her useful suggestion to Section 2. 


\section{Properties of ideals $\mathfrak{b}(\chi)$.}

For $\alpha \in K$ and each $\chi \in \hat{G}$, we define the resolvent of $\alpha$ with values in $K(\chi)$ by

$$
\langle\alpha, \chi\rangle=\langle\alpha, \chi\rangle_{K / k}:=\sum_{s \in G} \chi\left(s^{-1}\right) \alpha^{s} .
$$

For each $\chi \in \hat{G}$, let $L(\chi)$ be the $\mathfrak{o}_{k(\chi)}(S)$-module of rank one generated by all $\langle\alpha, \chi\rangle$ with $\alpha \in \mathfrak{o}_{K}(S)$. Let $\beta \in \mathfrak{o}_{K}$ be a free generator of $K$ over $k G$. Then there exists a fractional ideal $\mathfrak{b}(\chi)$ of $\mathfrak{o}_{k(\chi)}(S)$ such that

$$
L(\chi)=\mathfrak{b}(\chi)\langle\beta, \chi\rangle
$$

and we have

$$
\mathfrak{b}\left(\chi^{\omega}\right)=\mathfrak{b}(\chi)^{\omega},
$$

for all $\omega \in \operatorname{Gal}(k(\chi) / k)$, where we define $\chi^{\omega}(s):=\chi(s)^{\omega}$ for each $s \in G$ so that $\chi^{\omega} \in \hat{G}$.

In [8], we have chosen $\beta \in \mathfrak{v}_{K}$ such that $1 \otimes \beta$ is a free generator of $\mathfrak{o}_{\boldsymbol{k}_{\mathrm{p}}} \otimes_{\mathfrak{o}_{k}} \mathfrak{o}_{K}$ over $\mathfrak{o}_{k_{\mathfrak{p}}} G$ for each prime ideal $\mathfrak{p}$ of $\mathfrak{o}_{k}$, dividing the order of $G$ and not belonging to $S$. Then we have proved that $\mathfrak{o}_{K}(S) / \mathfrak{o}_{k}(S)$ has a normal basis if and only if for each $\chi \in \hat{G}, \mathfrak{b}(\chi)$ (depending on this $\beta$ ) is a principal ideal of $\mathfrak{o}_{k(\chi)}(S)$ and its generators satisfy some congruence conditions and some conditions (as in (2.2)) for Galois actions (See [8, Theorem 2.10 and Remark 2.11]). We have to use these results in this paper. In this section, we study the properties of these ideals in the ramified case (For the unramified case, see [8, Lemma 3.2]).

Let $g=g_{\chi}$ be the order of $\chi$ in $\hat{G}$ and $a(\chi)$ the module generated by the products $\prod_{i=1}^{g} \alpha_{i}$ with $\alpha_{i} \in L(\chi)$ so that $\mathfrak{a}(\chi)$ is an ideal of $\mathfrak{o}_{k(\chi)}(S)$ and it follows from (2.1) that

$$
\left(\langle\beta, \chi\rangle^{g_{\chi}}\right) \mathfrak{o}_{k(\chi)}(S)=\mathfrak{a}(\chi) \mathfrak{b}(\chi)^{-g_{\chi}} .
$$

Let $V(\chi)$ be the one dimensional $k(\chi)$-vector space of elements $\alpha$ of $K(\chi)$ with $\alpha^{s}=\chi(s) \alpha$ for all $s \in G a l(K(\chi) / k(\chi)) \hookrightarrow G$. Let $\widetilde{L}(\chi):=V(\chi) \cap \mathfrak{o}_{K(\chi)}(S)$ so that this is also a $\mathfrak{o}_{k(\chi)}(S)$ module of rank one. Therefore there exists a fractional ideal $\mathfrak{b}(\chi)$ of $\mathfrak{o}_{k(\chi)}(S)$ such that $\tilde{L}(\chi)=\tilde{\mathfrak{b}}(\chi)\langle\beta, \chi\rangle$. Similarly we define an ideal $\tilde{\mathfrak{a}}(\chi)$ of $\mathfrak{o}_{k(\chi)}(S)$. Then the formulas $(2.2)$ and (2.3) for these also hold. Since $\mathfrak{b}(\chi) \subset \tilde{b}(\chi)$, there exists an ideal $\mathfrak{c}(\chi)$ of $\mathfrak{o}_{k(\chi)}(S)$ such that

$$
\mathfrak{b}(\chi)=\widetilde{b}(\chi) \mathfrak{c}(\chi) \text {. }
$$

Now we consider the gap $\mathfrak{c}(\chi)$ between $\mathfrak{b}(\chi)$ and $\mathfrak{b}(\chi)$ and it gives a position of $\mathfrak{b}(\chi)$ in the decomposition (2.3) of a resolvent into ideals (See Proposition 2.1, Example 2.2 and Proposition 2.3). It follows from (2.4) and the formulas (2.3) for $L(\chi)$ and $\tilde{L}(\chi)$ that $\mathfrak{a}(\chi)=\tilde{\mathfrak{a}}(\chi) \mathfrak{c}(\chi)^{g}$. Let $\mathfrak{f}(\chi)$ be the Artin conductor of $\chi$ in $K / k$ which is an ideal of $\mathfrak{o}_{\boldsymbol{k}}$. By Fröhlich's result, $L(\chi) L(\bar{\chi})=\mathfrak{f}(\chi)$, where let $\bar{\chi}:=\chi^{-1}$ (See $[8$, Lemma 3.1]), hence $\mathfrak{a}(\chi) \mathfrak{a}(\bar{\chi})=\mathfrak{f}(\chi)^{g}$. So, 


$$
\tilde{\mathfrak{a}}(\chi) \tilde{\mathfrak{a}}(\bar{\chi})\{\mathfrak{c}(\chi) \mathfrak{c}(\bar{\chi})\}^{g}=\mathfrak{f}(\chi)^{g} \mathbf{o}_{\boldsymbol{k}(\chi)}(S) .
$$

From now on, let $\chi$ be a non-trivial character of $G$ and $k_{\chi}$ the fixed field of Ker $\chi$ in $K / k$ so that $k_{\chi} / k$ is a cyclic extension of degree $g$. Let

$$
l=l_{\chi}:=\left[k_{\chi}(\chi): k(\chi)\right](>1)
$$

so that $l \mid g$. Recall that $k_{\chi}(\chi) / k(\chi)$ is a cyclic Kummer extension of degree $l$ with primitive element $\langle\beta, \chi\rangle$ (See [8, Section 3]). So there are an $l$-power free ideal $A_{\chi}$ and an ideal $B_{\chi}$ of $\mathfrak{o}_{k(x)}(S)$ such that

$$
\left(\langle\beta, \chi\rangle^{l}\right)_{\mathfrak{o}_{k(\chi)}}(S)=A_{\chi} B_{\chi}^{l} .
$$

Since $\tilde{a}(\chi)$ is $g$-power free by [8, Lemma 2.8, (i)], it follows from the formula (2.3) for $\tilde{L}(\chi)$ that

$$
\tilde{\mathbf{a}}(\chi)=A_{\chi}^{g / l} \quad\left(\tilde{\mathrm{b}}(\chi)^{-1}=B_{\chi}\right) .
$$

Let $\zeta$ be a fixed primitive $g$ th root of unity and $\Omega=\Omega_{\chi}:=\operatorname{Gal}(k(\chi) / k)$. Then there exists a group injection $\imath$ from $\Omega$ into $(\mathbf{Z} / g \mathbf{Z})^{\times}$such that

$$
\zeta^{\omega}=\zeta^{\imath(\omega)} \quad \text { for all } \omega \in \Omega \text {. }
$$

If $1<d \mid g$, we write $l_{d}$ for the composition of $l$ and the canonical quotient map $(\mathbf{Z} / g \mathbf{Z})^{\times} \rightarrow(\mathbf{Z} / d \mathbf{Z})^{\times}$. For each $\omega \in \Omega / \operatorname{Ker} l_{d}$, let $t_{d}(\omega)$ be the integer satisfying

$$
l_{d}(\omega)=t_{d}(\omega) \bmod d, \quad 0<t_{d}(\omega)<d,
$$

and put

$$
\theta:=\sum_{\omega \in \Omega} t_{g_{x}}(\omega) \omega^{-1},
$$

which is in $\mathbf{Z} \Omega$. As $k_{\chi}(\chi) / k$ is an abelian extension, $A_{\chi}^{\omega-t_{l}(\omega)}$ is the $l$ th power of a fractional ideal of $\mathfrak{o}_{k(x)}(S)$ for each $\omega \in \Omega$. Hence

$$
\operatorname{ord}_{\mathfrak{P}}\left(A_{\chi}\right)=\operatorname{ord}_{\mathfrak{P}} \omega\left(A_{\chi}^{\omega}\right) \equiv t_{l}(\omega) \operatorname{ord}_{\mathfrak{P}} \omega\left(A_{\chi}\right)(\bmod l),
$$

for any prime ideal $\mathfrak{B}$ of $\mathfrak{o}_{k(x)}$, not lying above $S$, and any $\omega \in \Omega$.

Definition. For a prime ideal $\mathfrak{p}$ of $\mathfrak{o}_{k}$, we denote by $e_{\mathfrak{p}}$ and $Z_{\mathfrak{p}}$ the ramification index and the decomposition group of $\mathfrak{p}$ in $k(\chi) / k$ respectively. Let $\mathscr{U}=\mathscr{U}_{\chi}$ be the set of prime ideals of $\mathrm{o}_{k}$, not belonging to $S$, which are ramified in $k_{\chi} / k$, and $\mathscr{V}=\mathscr{V}_{x}$ the set of prime ideals $\mathfrak{p}$ of $\mathfrak{o}_{k}$, not belonging to $S$, such that $\mathfrak{P} \mid \mathfrak{p}$ and $\mathfrak{P} \mid A_{\chi}$ with some prime ideal $\mathfrak{P}$ of $\mathfrak{o}_{\boldsymbol{k}(x)}$.

We claim that $\mathscr{V} \subset \mathscr{U}$. If $\mathfrak{p} \in \mathscr{V}$, then $\mathfrak{P} \mid \mathfrak{p}$ and $\mathfrak{P} \mid A_{\chi}$ with some prime ideal $\mathfrak{P}$ of $\mathfrak{o}_{k(\chi)}$. Since $A_{\chi}$ is $l$-power free, $\mathfrak{P}$ is ramified in $k_{\chi}(\chi) / k(\chi)$. Therefore $\mathfrak{p}$ is ramified in $k_{\chi} / k$ so that $\mathfrak{p} \in \mathscr{U}$. Next we claim that 


$$
\tilde{\mathfrak{a}}(\chi) \tilde{\mathfrak{a}}(\bar{\chi})=\prod_{\mathfrak{p} \in \mathscr{V}} \prod_{\omega \in \Omega / Z_{\mathfrak{p}}} \mathfrak{P}^{g \omega},
$$

where $\mathfrak{P}$ is any prime ideal of $\mathfrak{o}_{k(\chi)}$ lying above $\mathfrak{p}$. By (2.7) and noting that $l=l_{\chi}=l_{\bar{\chi}}$, it is sufficient to prove that

$$
A_{\chi} A_{\bar{\chi}}=\prod_{\mathfrak{p} \in \mathscr{V}} \prod_{\omega \in \Omega / Z_{\mathfrak{p}}} \mathfrak{P}^{l \omega} .
$$

This is equivalent to the three statements: for all prime ideals $\mathfrak{P}$ of $\mathfrak{o}_{k(x)}$, not lying above $S$,

$$
\begin{gathered}
\operatorname{ord}_{\mathfrak{P}}\left(A_{\chi} A_{\bar{\chi}}\right)=0 \text { or } l, \\
\operatorname{ord}_{\mathfrak{P}}\left(A_{\chi} A_{\bar{\chi}}\right)>0 \Longrightarrow \forall \omega \in \Omega: \operatorname{ord}_{\mathfrak{P}}\left(A_{\chi} A_{\bar{\chi}}\right)>0, \\
\mathscr{V}=\left\{\mathfrak{P} \cap k \mid \mathfrak{P} \text { is a prime divisor of } A_{\chi} A_{\bar{\chi}}\right\} .
\end{gathered}
$$

It follows from (2.6) for $\chi$ and for $\bar{\chi}$ that

$$
A_{\chi} A_{\bar{\chi}}=\left(\langle\beta, \chi\rangle\langle\beta, \bar{\chi}\rangle B_{\chi}^{-1} B_{\bar{\chi}}^{-1}\right)^{l} .
$$

Since $\langle\beta, \chi\rangle\langle\beta, \bar{\chi}\rangle$ is in $k(\chi)$ (Cf. [8, Lemma 2.3, (iv)]), we have

$$
l \mid\left(\operatorname{ord}_{\mathfrak{P}}\left(A_{\chi}\right)+\operatorname{ord}_{\mathfrak{P}}\left(A_{\bar{\chi}}\right)\right) .
$$

So the fact that $A_{\chi}$ and $A_{\bar{\chi}}$ are $l$-power free implies (2.12) and also that

$$
\operatorname{ord}_{\mathfrak{P}}\left(A_{\chi}\right)>0 \Longleftrightarrow \operatorname{ord}_{\mathfrak{P}}\left(A_{\chi} A_{\bar{\chi}}\right)>0 \text {. }
$$

It follows from (2.9) that $\operatorname{ord}_{\mathfrak{P}}\left(A_{\chi}\right)>0 \Rightarrow \operatorname{ord}_{\mathfrak{P}} \omega\left(A_{\chi}\right)>0$. This fact, together with $(2.15)$ for $\mathfrak{P}$ and for $\mathfrak{P}^{\omega}$, gives (2.13). (2.14) follows from (2.15) and the definition of $\mathscr{V}$. Thus we have proved the claim (2.11), hence (2.10). By the definition of Artin conductors, $f(\chi)$ becomes the Artin conductor of the character of $G a l\left(k_{\chi} / k\right)$ associated with $\chi$. So by the assumed tameness outside $S$,

$$
\mathfrak{f}(\chi) \mathfrak{o}_{k}(S)=\prod_{\mathfrak{p} \in \mathscr{U}} \mathfrak{p}
$$

By the definition of $e_{\mathfrak{p}}$ and $Z_{\mathfrak{p}}$, we have $\mathfrak{p}=\prod_{\omega \in \Omega / Z_{\mathfrak{p}}} \mathfrak{P}^{e_{\mathfrak{p}} \omega}$. Hence (2.5), (2.10) and (2.16) yield the following proposition:

Proposition 2.1. Let $\beta \in \mathfrak{v}_{K}$ be a free generator of $K$ over $k G$ and $\chi(\neq 1) \in \hat{G}$. Let the ideal $\mathrm{c}(\chi)$ of $\mathrm{o}_{k(\chi)}(S)$ be as in (2.4). Then under the above notations, we have

$$
\mathfrak{c}(\chi) \mathfrak{c}(\bar{\chi})=\prod_{\mathfrak{p} \in \mathscr{U}_{\chi}-\mathscr{V}_{\chi}} \mathfrak{p} \cdot \prod_{\mathfrak{p} \in \mathscr{V}_{\chi}}\left(\prod_{\omega \in \Omega_{\chi} / Z_{\mathfrak{p}}} \mathfrak{P}^{\omega}\right)^{\boldsymbol{e}_{\mathfrak{p}}-1},
$$

where $\mathfrak{P}$ is any prime ideal of $\mathfrak{p}_{k(x)}$ lying above $\mathfrak{p} \in \mathscr{V}_{\chi}$. In particular, if $\mathscr{U}_{\chi}=\varnothing$ or $k$ contains a primitive $g_{\chi}$ th root of unity (i.e., $k=k(\chi)$, therefore $\mathscr{U}_{\chi}=\mathscr{V}_{\chi}$ and $e_{\mathfrak{p}}=1$ for all $\mathfrak{p}$ in $\mathscr{V}_{\chi}$ ), 
then we have $\mathfrak{c}(\chi)=(1)$ so that $\mathfrak{b}(\chi)=\tilde{b}(\chi)$ and $\mathfrak{a}(\chi)=\tilde{\mathfrak{a}}(\chi)$.

EXAMPLE 2.2. We shall give an abelian extension $K / k$ with $\mathscr{V}_{\chi} \subsetneq \mathscr{U}_{\chi}$ for a certain $\chi$ in $\hat{G}$. Then we have $\mathrm{c}(\chi) \neq(1)$ by Proposition 2.1 so that $\mathfrak{b}(\chi) \neq \tilde{b}(\chi)$. Let $p_{1}, p_{2}$ be odd prime numbers such that $p_{2} \equiv 1 \bmod p_{1}$. Let $K$ be a subfield of $\mathbf{Q}\left(\zeta_{p_{1} p_{2}}\right)$ with $\mathbf{Q}\left(\zeta_{p_{2}}\right) \subset K$ and $\left[K: \mathbf{Q}\left(\zeta_{p_{2}}\right)\right]>1$ and $k$ the unique subfield of $\mathbf{Q}\left(\zeta_{p_{2}}\right)$ with $\left[\mathbf{Q}\left(\zeta_{p_{2}}\right): k\right]=p_{1}$. Assume that the set $S$ does not contain any prime ideal of $\mathrm{o}_{k}$ lying above $p_{1}$ or $p_{2}$. Let $F$ be the unique subfield of $\mathbf{Q}\left(\zeta_{p_{1}}\right)$ with $[F: \mathbf{Q}]=\left[K: \mathbf{Q}\left(\zeta_{p_{2}}\right)\right]$, so that $\operatorname{Gal}\left(K / \mathbf{Q}\left(\zeta_{p_{2}}\right)\right) \cong$ $G a l(F k / k) \cong G a l(F / Q)$. Let $\psi_{1}$ be a non-trivial character of $G a l(F k / k)$ of order $m$ and $\psi_{2}$ a character of $\operatorname{Gal}\left(\mathbf{Q}\left(\zeta_{p_{2}}\right) / k\right)$ of order $p_{1}$. Let $\chi$ be the character of $G$ corresponding to $\left(\psi_{1}, \psi_{2}\right)$ by the canonical isomorphism:

$$
\hat{G} \cong \operatorname{Gal}(F k / k)^{\wedge} \times \operatorname{Gal}\left(\mathbf{Q}\left(\zeta_{p_{2}}\right) / k\right)^{\wedge},
$$

so that $g_{\chi}=m p_{1}$ by $\left(p_{1}, m\right)=1$. Since $\left(p_{2}, m p_{1}\right)=1$, we have $k \cap \mathbf{Q}(\chi)=\mathbf{Q}$. Also $\mathbf{Q}\left(\zeta_{p_{2}}\right) \subset$ $k_{\chi} \subset K,\left[k_{\chi}: \mathbf{Q}\left(\zeta_{p_{2}}\right)\right]=m$. Therefore

$$
\mathscr{U}=\left\{\mathfrak{p} ; \text { prime in } \mathfrak{o}_{k} ; \mathfrak{p} \mid p_{1} \text { or } \mathfrak{p} \mid p_{2}\right\} .
$$

Since a prime ideal of $\mathrm{o}_{k(x)}$ lying above $p_{2}$ is the only ramified ideal in $k_{x}(\chi) / k(\chi)$ and it is tamely ramified, a prime divisor of the ideal $A_{\chi}$ divides $p_{2}$. Hence

$$
\mathscr{V}=\left\{\mathfrak{p} ; \text { prime in } \mathfrak{o}_{k} ; \mathfrak{p} \mid p_{2}\right\} .
$$

So $\mathscr{V} \subsetneq \mathscr{U}$. (Note that $e_{\mathfrak{p}}=1$ for all $\mathfrak{p}$ in $\mathscr{V}$ now.)

The following proposition is a generalization of Sodaïgui [9, Théorème 2.2]:

Proposition 2.3. Let $\beta, \chi$ be as in Proposition 2.1 and $\mathrm{b}(\chi)($ resp. $\mathrm{a}(\chi))$ a fractional ideal of $\mathbf{o}_{k(x)}(S)$ depending on $\beta$ as in (2.1) (resp. (2.3)). Suppose that $\mathscr{U}_{\chi} \neq \varnothing$.

(i) Assume that (A1): $\mathfrak{p} \in \mathscr{U}_{\chi} \Rightarrow \mathfrak{p} \nmid g_{\chi}$. Then $\mathfrak{a}(\chi)$ is $g_{\chi}$-power free.

(ii) Assume that the map 1 is an isomorphism (i.e., $k \cap \mathbf{Q}(\chi)=\mathbf{Q})$ and (A2): for all $\mathfrak{p}$ in $\mathscr{U}_{\chi}, \mathfrak{p}$ is totally ramified in $k_{\chi} / k$. Then any $\mathfrak{p}$ in $\mathscr{U}_{\chi}$ is completely decomposed in $k(\chi) / k$ and we have

$$
\left(\langle\beta, \chi\rangle^{\boldsymbol{g}_{\boldsymbol{x}}}\right) \mathfrak{\mathfrak { o }}_{\boldsymbol{k}(\chi)}(S)=\prod_{\mathfrak{p} \in \mathscr{U}_{x}} \mathfrak{P}^{\theta} \mathfrak{b}(\chi)^{-\boldsymbol{g}_{\boldsymbol{\chi}}},
$$

where $\mathfrak{P}$ is some prime ideal of $\mathfrak{o}_{k(x)}$ lying above $\mathfrak{p}$ and $\theta$ is defined in (2.8).

REMARK 2.4. If $g_{\chi}$ is a prime power, then the assumption (A1) holds, because any $\mathfrak{p}$ in $\mathscr{U}$ is tamely ramified in $k_{\chi} / k$.

Proof of Proposition 2.3. (i) By (A1), we have $e_{\mathfrak{p}}=1$ for all $\mathfrak{p} \in \mathscr{U}$. Let $\mathfrak{p} \in \mathscr{U}$ and $\mathfrak{P}$ be a prime ideal of $\mathfrak{o}_{k(x)}$ with $\mathfrak{P} \mid \mathfrak{p}$. Since $e_{\mathfrak{p}}=1, \mathfrak{P}$ is ramified in $k_{\chi}(\chi) / k(\chi)$. Also $\mathfrak{P} \nmid l$. Therefore by Kummer theory, $\mathfrak{P} \mid A_{\chi}$ so that $\mathfrak{p} \in \mathscr{V}$. Thus $\mathscr{U}=\mathscr{V}$. Hence $\mathfrak{c}(\chi)=(1)$ by Proposition 2.1. So by $(2.4), \mathfrak{b}(\chi)=\mathfrak{b}(\chi)$ so that $\mathfrak{a}(\chi)=\tilde{a}(\chi)$. This proves the assertion (i).

(ii) By (A2), the assumption (A1) holds so that the assertion (i) is true. For $\mathfrak{p} \in \mathscr{U}$, 
since $e_{\mathfrak{p}}=1$ and $\mathfrak{p}$ is totally ramified, we have $k_{\chi} \cap k(\chi)=k$, therefore $l=g$. Consequently $\mathfrak{a}(\chi)=\tilde{a}(\chi)=A_{\chi}$ by (2.7). We define a subset $\mathscr{V}_{1}$ of $\mathscr{V}$ by

$$
\mathscr{V}_{1}:=\left\{\mathfrak{P} \cap k \mid \mathfrak{P} \text { is a prime ideal of } \mathfrak{o}_{k(\chi)} \text { with } \operatorname{ord}_{\mathfrak{P}}\left(A_{\chi}\right)=1\right\} \text {. }
$$

Claim that $\mathscr{U}=\mathscr{V}_{1}$. Let $\mathfrak{p} \in \mathscr{U}$ and $\mathfrak{P}$ be a prime ideal of $\mathfrak{o}_{k(x)}$ with $\mathfrak{P} \mid \mathfrak{p}$. Then $i:=$ $\operatorname{ord}_{\mathfrak{P}}\left(A_{\chi}\right) \geq 1$ (i.e., $\mathfrak{p} \in \mathscr{V}$ ) as seen above. Since $g$ is the ramification index of $\mathfrak{P}$ in $k_{\chi}(\chi) / k(\chi)$, we have $g=g /(i, g)$ from Kummer theory $([3$, p. 92]). So $(i, g)=1$. Since $\imath$ is surjective, there is some $\omega$ in $\Omega$ such that $i=t_{g}(\omega)$, therefore $1 \equiv t_{g}\left(\omega^{-1}\right) i \equiv \operatorname{ord}_{\mathfrak{P}^{\omega}\left(A_{\chi}\right)}$ $\bmod g$ by (2.9). As $0<\operatorname{ord}_{\mathfrak{P}^{\omega}}\left(A_{\chi}\right)<g$, we have $\operatorname{ord}_{\mathfrak{P}^{\omega}}\left(A_{\chi}\right)=1$. Hence $\mathfrak{p}=\mathfrak{P}^{\omega} \cap k \in \mathscr{V}_{1}$. Thus $\mathscr{U}=\mathscr{V}_{1}$. For $\mathfrak{p} \in \mathscr{U}$, let $\omega \in Z_{\mathfrak{p}}$ and $\mathfrak{P}$ a prime ideal of $\mathfrak{o}_{k(\chi)}$ with $\mathfrak{P} \mid \mathfrak{p}$. Since $\mathfrak{P}^{\omega}=\mathfrak{P}$ and $\mathfrak{p} \in \mathscr{V}_{1}, 1 \equiv t_{g}(\omega) \bmod g$ by (2.9), therefore $\omega=1 ; \mathfrak{p}$ is completely decomposed in $k(\chi) / k$. Since $\mathscr{U}=\mathscr{V}_{1}$, we can define a square free ideal $C$ of $\mathfrak{o}_{k(\chi)}(S)$ by $C:=\prod_{\mathfrak{p} \in \mathscr{U}} \mathfrak{P}^{\omega}$, where $\mathfrak{P}$ is some prime ideal of $\mathfrak{o}_{k(\chi)}$ with $\mathfrak{P} \mid \mathfrak{p}$ and $\operatorname{ord}_{\mathfrak{P}}\left(A_{\chi}\right)=1$. Then (2.9) and the assumption that $\imath$ is surjective imply $A_{\chi}=C^{\theta}$. Thus the assertion (ii) is proved.

\section{Decomposition of prime ideals.}

In this section, suppose that $k$ is a totally real number field or a $C M$-field. Let $l$ be an odd prime or $l=4$, and $\mathfrak{p}$ a prime ideal of $\mathfrak{o}_{k}$ such that $\mathfrak{p} \nmid l$. We assume that

$$
k / \mathbf{Q} \text { is Galois and } F:=k \cap \mathbf{Q}\left(\zeta_{l}\right) \subset k^{+},
$$

so that $k / F$ is Galois and $F$ is totally real. Since $l$ is an odd prime or $l=4, \operatorname{Gal}\left(\mathbf{Q}\left(\zeta_{l}\right) / F\right)$ is cyclic. By $\mathfrak{p} \nmid l, \mathfrak{p} \cap \mathfrak{o}_{F}$ is unramified in $\mathbf{Q}\left(\zeta_{l}\right) / F$. Now we wish to discuss the following problem:

(\#): For any prime ideal $\mathfrak{P}$ of $\mathfrak{o}_{k\left(\zeta_{l}\right)}$ with $\mathfrak{P} \mid \mathfrak{p}, \mathfrak{P}$ is not decomposed in $k\left(\zeta_{l}\right) / k\left(\zeta_{l}\right)^{+} ?$

So we need the following proposition:

Proposition 3.1. Let $F$ be a totally real number field and $K_{i} / F(i=1,2)$ a finite Galois extension with Galois group $G_{i}$ such that $K_{1} \cap K_{2}=F$. Assume that $K_{1}$ is a totally real number field or a $C M$-field, and $K_{2}$ is a CM-field, so that $\left|G_{2}\right|>1$. Suppose that $G_{2}$ has only an element of order two (For example, this is true when $G_{2}$ is cyclic). Put $L:=K_{1} K_{2}$ which is a CM-field. Let $\mathfrak{B}$ be a prime ideal of $\mathfrak{o}_{L}, \mathfrak{p}_{i}:=\mathfrak{P} \cap \mathfrak{o}_{K_{i}}(i=1,2)$ and $p:=\mathfrak{B} \cap \mathfrak{D}_{F}$. Suppose that $p$ is unramified in $K_{2} / F . f_{i}(i=1,2)$ denotes the residue degree of $p$ in $K_{i} / F$. Then we have the following:

(I) The case where $K_{1}$ is totally real.

$\mathfrak{P}$ is not decomposed in $L / L^{+} \Leftrightarrow \operatorname{ord}_{2}\left(f_{1}\right)+1 \leq \operatorname{ord}_{2}\left(f_{2}\right)$.

(II) The case where $K_{1}$ is a CM-field.

(i) If $\mathfrak{p}_{1}$ is decomposed in $K_{1} / K_{1}^{+}$, then $\mathfrak{P}$ is decomposed in $L / L^{+}$.

(ii) If $\mathfrak{p}_{1}$ is ramified in $K_{1} / K_{1}^{+}$, then $\mathfrak{P}$ is not decomposed in $L / L^{+} \Leftrightarrow \operatorname{ord}_{2}\left(f_{1}\right)+1 \leq$ 
$\operatorname{ord}_{2}\left(f_{2}\right)$.

(iii) If $\mathfrak{p}_{1}$ is inert in $K_{1} / K_{1}^{+}$, then $\mathfrak{P}$ is not decomposed in $L / L^{+} \Leftrightarrow \operatorname{ord}_{2}\left(f_{1}\right)=\operatorname{ord}_{2}\left(f_{2}\right)$ $(>0)$.

Proof. Let $\sigma_{i}(i=1,2)$ be a Frobenius automorphism of $\mathfrak{p}_{i}$ in $K_{i} / F$, and $T_{i}$ and $Z_{i}$ the inertia and decomposition groups of $\mathfrak{p}_{i}$ in $K_{i} / F$, respectively. Let $\theta$ be a Frobenius automorphism of $\mathfrak{P}$ in $L / F$, and $T$ and $Z$ the inertia and decomposition groups of $\mathfrak{B}$ in $L / F$, respectively. As $K_{1} \cap K_{2}=F, G a l(L / F)$ is identified with $G_{1} \times G_{2}$. Since $|T|=\left|T_{1}\right|$ by $T_{2}=\{1\}, T \subset T_{1} \times T_{2}$ implies $T=T_{1} \times\{1\}$. If $\theta_{i}(i=1,2)$ is the restriction of $\theta$ to $K_{i}$, then $\theta=\left(\theta_{1}, \theta_{2}\right)$ and $\theta_{i}$ is a Frobenius automorphism of $\mathfrak{p}_{i}$ in $K_{i} / F$. Therefore $\theta_{1} T_{1}=$ $\sigma_{1} T_{1}$ and furthermore $\theta_{2}=\sigma_{2}$ by $T_{2}=\{1\}$. Hence

$$
Z=\bigcup_{m} \theta^{m} T=\bigcup_{m}\left(\sigma_{1}, \sigma_{2}\right)^{m} \cdot\left(T_{1} \times\{1\}\right),
$$

where $m$ ranges over all integers. Let $\rho_{i}(i=1,2)$ be the restriction of the complex conjugation to $K_{i}$. Since $F$ is real, $\rho_{i} \in G_{i}$ and furthermore the order of $\rho_{2}$ in $G_{2}$ is two since $K_{2}$ is a $C M$-field. Let $H:=\left\langle\left(\rho_{1}, \rho_{2}\right)\right\rangle$, where note that $\rho_{1}=1$ when $K_{1}$ is real. Then $L^{+}$is the fixed field of $H$ in $L / F$. So,

$$
\mathfrak{P} \text { is not decomposed in } L / L^{+} \Longleftrightarrow H \subset Z \text {, }
$$

because $H \cap Z$ is the decomposition group of $\mathfrak{P}$ in $L / L^{+}$. If $\mathfrak{P}$ is not decomposed in $L / L^{+}$, then $\rho_{1} \in Z_{1}$ from (3.3) and $Z \subset Z_{1} \times Z_{2}$, so that $\left\langle\rho_{1}\right\rangle \cap Z_{1}=\left\langle\rho_{1}\right\rangle$, hence $\mathfrak{p}_{1}$ is not decomposed in $K_{1} / K_{1}^{+}$. This proves the assertion (II-i). For each $i=1,2$, let $t_{i}:=\operatorname{ord}_{2}\left(f_{i}\right)$.

The cases (I) and (II-ii). Since $\left\langle\rho_{1}\right\rangle \cap T_{1}$ is the inertia group of $\mathfrak{p}_{1}$ in $K_{1} / K_{1}^{+}, \mathfrak{p}_{1}$ is ramified in $K_{1} / K_{1}^{+} \Leftrightarrow \rho_{1} \in T_{1}$. So $\rho_{1} T_{1}=T_{1}$ by the assumptions. By (3.2) and (3.3), we may show that $t_{1}+1 \leq t_{2} \Leftrightarrow$ there exists an integer $m$ such that $T_{1}=\sigma_{1}^{m} T_{1}$ and $\rho_{2}=\sigma_{2}^{m}$. If such $m$ exists, then we have $f_{1} \mid m, f_{2}$ is even, $\left(f_{2} / 2\right) \mid m$ and $m /\left(f_{2} / 2\right)$ is odd, because $f_{1}$ is the order of $\sigma_{1} T_{1}$ in $Z_{1} / T_{1}$ and $f_{2}$ is the order of $\sigma_{2}$ in $G_{2}$. Let $a$ be the least common multiple of $f_{1}$ and $f_{2} / 2$. Since $a \mid m$, we have

$$
\operatorname{Max}\left(t_{1}, t_{2}-1\right)=\operatorname{ord}_{2}(a) \leq \operatorname{ord}_{2}(m)=\operatorname{ord}_{2}\left(f_{2} / 2\right)=t_{2}-1 \text {. }
$$

Therefore $t_{1}+1 \leq t_{2}$. Conversely, assume that this holds. So $f_{2}$ is even. Let $a$ be the same meaning as above. Then $T_{1}=\sigma_{1}^{a} T_{1}$. Since $t_{1} \leq t_{2}-1, \operatorname{ord}_{2}(a)=\operatorname{ord}_{2}\left(f_{2} / 2\right)$ so that the order of $\sigma_{2}^{a}$ is two. Since $G_{2}$ has only an element of order two, we have $\rho_{2}=\sigma_{2}^{a}$. This proves the assertions.

The case (II-iii). Now the order of $\rho_{1}$ in $G_{1}$ is two. Since $\mathfrak{p}_{1}$ is inert in $K_{1} / K_{1}^{+}$, $t_{1}>0,\left\langle\rho_{1}\right\rangle \cap Z_{1}=\left\langle\rho_{1}\right\rangle$ and $\left\langle\rho_{1}\right\rangle \cap T_{1}=\{1\}$. So $\rho_{1} \in Z_{1}$ and $\rho_{1} \notin T_{1}$. Therefore $\rho_{1} T_{1}$ is the element of order two in the cyclic group $Z_{1} / T_{1}$. By (3.2) and (3.3), we may show that $t_{1}=t_{2} \Leftrightarrow$ there exists an integer $m$ such that $\rho_{1} T_{1}=\sigma_{1}^{m} T_{1}$ and $\rho_{2}=\sigma_{2}^{m}$. This is similarly proved as in the above cases (e.g., let $a$ be the least common multiple of $f_{1} / 2$ 
and $f_{2} / 2$ in this case).

Return to the situation as before Proposition 3.1. Considering (II-i) of its proposition, we distinguish two cases:

(C1) $k$ is totally real or " $k$ is a $C M$-field and $\mathfrak{p}$ is ramified in $k / k^{+}$".

(C2) $k$ is a $C M$-field and $\mathfrak{p}$ is inert in $k / k^{+}$.

Let $p:=\mathfrak{p} \cap \mathbf{Z}$. We denote by $a$ and $b$ the residue degrees of $p$ in $k / \mathbf{Q}$ and $\mathbf{Q}\left(\zeta_{l}\right) / \mathbf{Q}$, respectively. Let $f, f_{1}$ and $f_{2}$ be the residue degrees of $\mathfrak{p} \cap \mathfrak{o}_{F}$ in $F / \mathbf{Q}, k / F$ and $\mathbf{Q}\left(\zeta_{l}\right) / F$, respectively. So $a=f f_{1}, b=f f_{2}$, therefore

$$
\operatorname{ord}_{2}(a)=\operatorname{ord}_{2}(f)+\operatorname{ord}_{2}\left(f_{1}\right), \quad \operatorname{ord}_{2}(b)=\operatorname{ord}_{2}(f)+\operatorname{ord}_{2}\left(f_{2}\right) .
$$

Note that $F=\mathbf{Q}, a=f_{1}$ and $b=f_{2}$ hold under the assumption (3.1) when $l=4$.

LEMMA 3.2. Let $l$ be an odd prime or $l=4$, and $\mathfrak{p}$ a prime ideal of $\mathfrak{o}_{k}$ such that $\mathfrak{p} \nmid l$. Put $\mathrm{Np}:=\left|\mathfrak{o}_{k} / \mathfrak{p}\right|$. Then under the assumption (3.1) and the above notations, we have

(i) If $l$ is an odd prime and $l \mid(\mathrm{Np}-1)$, then (\#) does not hold in the case (C1).

(ii) When $l=4,(\#)$ holds $\Leftrightarrow \mathrm{Np} \equiv 3 \bmod 4$ in the case $(\mathrm{C1})$, and "ord ${ }_{2}(a)=1$ and $p \equiv 3 \bmod 4$ " in the case (C2).

Proof. (i) By $l \mid(\mathbf{N p}-1), p^{a}=\mathbf{N p} \equiv 1 \bmod l$. Since $b$ is the order of $p \bmod l$, we have $b \mid a$ so that $\operatorname{ord}_{2}(b) \leq \operatorname{ord}_{2}(a)$. It follows from (3.4) that $\operatorname{ord}_{2}\left(f_{2}\right) \leq \operatorname{ord}_{2}\left(f_{1}\right)<$ $\operatorname{ord}_{2}\left(f_{1}\right)+1$. Hence (\#) does not hold by Proposition 3.1, (I), (II-ii) (more precisely, any prime ideal $\mathfrak{P}$ of $\mathfrak{o}_{\boldsymbol{k}\left(\zeta_{l}\right)}$ with $\mathfrak{P} \mid \mathfrak{p}$ is decomposed in $\left.k\left(\zeta_{l}\right) / k\left(\zeta_{l}\right)^{+}\right)$.

(ii) Now $\mathbf{Q}\left(\zeta_{l}\right)=\mathbf{Q}(\sqrt{-1})$ and $p$ is an odd prime. So,

$$
\begin{aligned}
& p \equiv 1 \bmod 4 \Leftrightarrow p \text { is decomposed in } \mathbf{Q}\left(\zeta_{l}\right) / \mathbf{Q} \Leftrightarrow b=1 \Leftrightarrow \operatorname{ord}_{2}(b)=0, \\
& p \equiv 3 \bmod 4 \Leftrightarrow p \text { is inert in } \mathbf{Q}\left(\zeta_{l}\right) / \mathbf{Q} \Leftrightarrow b=2 \Leftrightarrow \operatorname{ord}_{2}(b)=1 .
\end{aligned}
$$

Hence $\operatorname{ord}_{2}(a)+1=(\leq) \operatorname{ord}_{2}(b) \Leftrightarrow " p \equiv 3 \bmod 4$ and $\operatorname{ord}_{2}(a)=0 " \Leftrightarrow \mathrm{Np}=p^{a} \equiv 3 \bmod 4$. In (C2), we have $\operatorname{ord}_{2}(a)>0$. Since $\operatorname{ord}_{2}(b) \leq 1, \operatorname{ord}_{2}(a)=\operatorname{ord}_{2}(b) \Leftrightarrow \operatorname{ord}_{2}(a)=1$ and $p \equiv 3$ mod 4. Now the assertions follow from Proposition 3.1.

For a prime ideal $\mathfrak{p}$ of $\mathfrak{o}_{k}$ with $\mathfrak{p} \nmid l$, putting $p:=\mathfrak{p} \cap \mathbf{Z}$, let $a_{\mathfrak{p}}$ (resp. $b_{\mathfrak{p}}$ ) be the residue degree of $p$ in $k / \mathbf{Q}\left(\operatorname{resp} . \mathbf{Q}\left(\zeta_{l}\right) / \mathbf{Q}\right)$. When $l$ is an odd prime (resp. $l=4$ ), we define the sets of prime ideals of $\mathfrak{o}_{k}$ as follows.

$$
\mathfrak{\Im}_{1, l}:=\left\{\mathfrak{p} \mid \mathfrak{p} \nmid l \text { and } \operatorname{ord}_{2}\left(a_{\mathfrak{p}}\right)+1 \leq \operatorname{ord}_{2}\left(b_{\mathfrak{p}}\right)(\operatorname{resp} . \mathrm{Np} \equiv 3 \bmod 4)\right\},
$$

if $k$ is totally real, and

$$
\begin{aligned}
& \mathfrak{S}_{21, l}:=\left\{\mathfrak{p} \mid \mathfrak{p} \nmid l, \mathfrak{p} \text { is ramified in } k / k^{+}\right. \text {and } \\
&\left.\quad \operatorname{ord}_{2}\left(a_{\mathfrak{p}}\right)+1 \leq \operatorname{ord}_{2}\left(b_{\mathfrak{p}}\right)(\operatorname{resp} . \mathrm{Np} \equiv 3 \bmod 4)\right\}, \\
& \mathfrak{S}_{22, l}:=\left\{\mathfrak{p} \mid \mathfrak{p} \nmid l, \mathfrak{p} \text { is inert in } k / k^{+}\right. \text {and } \\
&\left.\quad \operatorname{ord}_{2}\left(a_{\mathfrak{p}}\right)=\operatorname{ord}_{2}\left(b_{\mathfrak{p}}\right)\left(\operatorname{resp} . \operatorname{ord}_{2}\left(a_{\mathfrak{p}}\right)=1 \text { and } p \equiv 3 \bmod 4\right)\right\},
\end{aligned}
$$


if $k$ is a $C M$-field. Then Proposition 3.1 and Lemma 3.2, (ii) yield:

Proposition 3.3. Let $l$ be an odd prime or $l=4$. Under the above notations and the assumption (3.1), suppose that $S$ is a subset of the set $\mathfrak{\Xi}_{1, l}\left(\right.$ resp. $\left.\mathfrak{S}_{21, l} \cup \mathfrak{S}_{22, l}\right)$, if $k$ is totally real (resp. a CM-field). Then for any prime ideal $\mathfrak{P}$ of $\mathfrak{v}_{k\left(\zeta_{l}\right)}$ lying above $S, \mathfrak{P}$ is not decomposed in $k\left(\zeta_{l}\right) / k\left(\zeta_{l}\right)^{+}$.

Now we discuss the cardinal of sets $\mathfrak{S}_{1, l}$ and $\mathfrak{S}_{22, l}$.

LEMMA 3.4. Let $l$ be an odd prime and $e:=\operatorname{ord}_{2}(l-1)(\geq 1)$. For a prime $p$ such that $p \nmid l$, let $b_{p}$ be the residue degree of $p$ in $\mathbf{Q}\left(\zeta_{l}\right) / \mathbf{Q}$. Then for each $i(1 \leq i \leq e)$, there are infinitely many primes $p$ such that $i=\operatorname{ord}_{2}\left(b_{p}\right)$ and $p \nmid l$.

Proof. Let $r$ be a primitive root $\bmod l$ and $c$ a divisor of $(l-1) / 2^{e}$. By Dirichlet's density theorem, there are infinitely many primes $p$ such that

$$
p \equiv r^{2^{e-i}} \bmod l \text {. }
$$

For such primes $p$, we have $b_{p}=(l-1) /\left(2^{e-i} c\right)$, therefore $i=\operatorname{ord}_{2}\left(b_{p}\right)$. This proves our lemma.

LEMMA 3.5. Let $l$ be an odd prime or $l=4$. Assume that $k / Q$ is an abelian extension with the discriminant $d$. Then under the above notations, we have

(i) $\left[k\right.$ : Q] is not a power of 2 and $(d, l)=1 \Rightarrow\left|\Im_{1, l}\right|=\infty$.

(ii) $l \equiv 1 \bmod 4$ and $(d, l)=1 \Rightarrow\left|\Im_{1, l}\right|=\infty$.

(iii) Let $k$ be a CM-field. If we put

$$
\mathfrak{S}_{2, l}:=\left\{\mathfrak{p} \mid \mathfrak{p} \text { is inert in } k / k^{+} \text {and } p^{a_{\mathfrak{p}} / 2} \equiv-1 \bmod l\right\},
$$

then $\mathfrak{S}_{2, l} \subset \mathfrak{S}_{22, l}$ and $\left|\mathfrak{S}_{2, l}\right|=\infty$.

ProOF. (i) Since $[k: \mathbf{Q}]$ is not a power of 2 , there is an element $\sigma$ of $\operatorname{Gal}(k / \mathbf{Q})$ of odd prime order. By Tchebotarev's density theorem, there are infinitely many primes $p_{0}$ with $p_{0} \nmid d$, whose Frobenius automorphism in $k / \mathbf{Q}$ is equal to $\sigma$. Take such a prime $p_{0}$. Let $m(\in \mathbf{Z})$ be the conductor of $k / \mathbf{Q}$ so that $G a l(k / \mathbf{Q})$ is isomorphic to the quotient group of $(Z / m Z)^{\times}$. Since $(d, l)=1,(m, l)=1$. By Dirichlet's density theorem, there are infinitely many primes $p$ such that $p \equiv p_{0} \bmod m$, and (3.5) for $i=1(\operatorname{resp} . p \equiv 3 \bmod 4)$ holds if $l$ is odd (resp. $l=4$ ). Let $\mathfrak{p}$ be a prime ideal of $\mathfrak{o}_{k}$ lying above such a prime $p$. Then $\operatorname{ord}_{2}\left(b_{\mathfrak{p}}\right)=1$ by Lemma 3.4 and the proof of Lemma 3.2, (ii). Furthermore $\operatorname{ord}_{2}\left(a_{\mathfrak{p}}\right)=0$, because $\sigma$ is also the Frobenius automorphism of $p$ in $k / \mathbf{Q}$ and $a_{\mathfrak{p}}$ is the order of $\sigma$. Hence $\mathfrak{p} \in \mathfrak{S}_{1, l}$. This proves the assertion.

(ii) Now $l$ is odd and, by (i), we may assume that $[k: \mathbf{Q}]$ is a power of 2 . So there is an element $\sigma$ of $\mathrm{Gal}(\mathrm{k} / \mathbf{Q})$ of order two. Then the same argument as in (i) proves the assertion. (Since $l \equiv 1 \bmod 4$, use (3.5) for $i=2$. Then we obtain $\operatorname{ord}_{2}\left(b_{\mathfrak{p}}\right)=2$, $\operatorname{ord}_{2}\left(a_{\mathrm{p}}\right)=1$.)

(iii) Let $\mathfrak{p} \in \mathcal{S}_{2, l}$. Since $b_{\mathfrak{p}}$ is the order of $p \bmod l$ and $p^{a_{\mathfrak{p}} / 2} \equiv-1 \bmod l$, we have 
$\operatorname{ord}_{2}\left(a_{\mathfrak{p}}\right)=\operatorname{ord}_{2}\left(b_{\mathfrak{p}}\right)\left(\operatorname{resp} . \operatorname{ord}_{2}\left(a_{\mathfrak{p}}\right)=1\right.$ and $\left.p \equiv 3 \bmod 4\right)$ when $l$ is odd (resp. $\left.l=4\right)$. Hence $\mathfrak{p} \in \mathfrak{S}_{22, l}$ so that $\mathfrak{S}_{2, l} \subset \mathfrak{S}_{22, l}$. By Dirichlet's density theorem, there are infinitely many primes $p$ such that $p \equiv-1 \bmod m l$. Let $\mathfrak{p}$ be a prime ideal of $\mathfrak{o}_{k}$ lying above such a prime $p$. Since $p \equiv-1 \bmod m$, the complex conjugation $\rho(\neq 1)$ on $k$ is the Frobenius automorphism of $p$ in $k / \mathbf{Q}$. So $a_{\mathfrak{p}}=2$ and $\mathfrak{p}$ is inert in $k / k^{+}$. Hence $\mathfrak{p} \in \mathfrak{S}_{2, l}$. This proves our lemma.

\section{A sufficient condition for the non-existence.}

We assume that $k / \mathbf{Q}$ is a Galois extension of even degree and $K / k$ is a finite abelian extension with conductor $m$. And we write the finite component $m_{0}$ of $m$ as the form $\mathfrak{m}_{0}=\mathfrak{m}_{1} \mathrm{~m}_{2}$, satisfying that " $\mathfrak{p} \mid \mathfrak{m}_{1} \Rightarrow \operatorname{ord}_{\mathfrak{p}}\left(\mathfrak{m}_{0}\right)=1$ " and " $\mathfrak{p} \mid \mathfrak{m}_{2} \Rightarrow \operatorname{ord}_{\mathfrak{p}}\left(\mathfrak{m}_{0}\right) \geq 2$ ". Let $l$ be a fixed odd prime such that $k \cap \mathbf{Q}\left(\zeta_{l}\right)=\mathbf{Q}$. Put $\Im_{l}:=\Im_{1, l}$ (resp. $\left.\Im_{21, l} \cup \Im_{22, l}\right)$, when $k$ is totally real (resp. a $C M$-field), where the set $\mathfrak{S}_{*, l}$ is defined before Proposition 3.3. Suppose that $S=S_{l}$ is a finite subset of $\mathcal{S}_{l}$ such that $\left\{\mathfrak{p} ; \mathfrak{p} \mid \mathfrak{m}_{2}\right\} \subset S$. So $S$ contains all prime ideals of $\mathfrak{o}_{k}$ which are wildly ramified in $K / k$, by the conductor-discriminant theorem. For a prime ideal $\mathfrak{p}$ of $\mathfrak{o}_{k}, e_{\mathfrak{p}}$ denotes the ramification index of $\mathfrak{p}$ in $k / \mathbf{Q}$. We define the finite set of prime ideals of $\mathfrak{p}_{k}$ as follows.

$$
\mathfrak{I}_{l}:=\left\{\mathfrak{p} ; 2 \mid e_{\mathfrak{p}}, \operatorname{ord}_{2}\left(b_{\mathfrak{p}}\right)=0\right\},
$$

where $b_{\mathfrak{p}}$ is the residue degree of $\mathfrak{p} \cap \mathbf{Z}$ in $\mathbf{Q}\left(\zeta_{l}\right) / \mathbf{Q}$. Then note that $\mathfrak{I}_{l} \cap \mathfrak{S}_{1, l}=\mathfrak{I}_{l} \cap$ $\varsigma_{21, l}=\varnothing$.

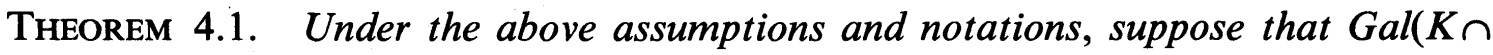
$\tilde{k} / k)$ is a 2-group and that there exists some $\mathfrak{p}$ with $\mathfrak{p} \nmid 2$ in $\mathfrak{I}_{l}$, not belonging to $S$, such that $l \mid[K \cap k(\mathfrak{p}): k]$. Then $\mathfrak{o}_{K}(S) / \mathfrak{o}_{k}(S)$ does not have a normal basis.

REMARK 4.2. As seen below, note that $\mathfrak{p} \nmid l$. And note that $l \mid[k(\mathfrak{p}): k]=(\mathrm{Np}-$ 1) $h_{k} / w_{\mathfrak{p}}$, where $w_{\mathfrak{p}}:=\left|\left(\mathfrak{o}_{k}^{\times}+\mathfrak{p}\right) / \mathfrak{p}\right|$ and $h_{k}:=[\tilde{k}: k]$.

ProOF of TheOREM 4.1. Let $L:=K \cap k(\mathfrak{p})$. Since $l \mid[L: k]$, there exists some $\chi$ in $\operatorname{Gal}(L / k)^{\wedge}$ such that $g_{\chi}=l$. Let $k_{\chi}$ be the fixed field of $\operatorname{Ker} \chi$ in $L / k$. Then $\mathfrak{p}$ is ramified in $k_{\chi} / k$. If not so, then $k_{\chi} \subset \tilde{k}$ so that $k_{\chi} \subset K \cap \tilde{k}$. This contradicts that $\operatorname{Gal}(K \cap \tilde{k} / k)$ is a 2-group. Consequently since $k_{\chi} \subset k(\mathfrak{p}), \mathfrak{p}$ is tamely ramified in $k_{\chi} / k$ so that $\mathfrak{p} \nmid g_{\chi}$.

Assume that $\mathfrak{o}_{K}(S) / \mathfrak{o}_{k}(S)$ has a normal basis; therefore so does $\mathfrak{o}_{L}(S) / \mathfrak{o}_{k}(S)$. By the assumed tameness in $K / k$ outside $S$, there is some $\gamma$ in $\mathfrak{o}_{L}(S)$ such that $\operatorname{Tr}_{L / k}(\gamma)=$ $\langle\gamma, 1\rangle_{L / k}=1$. This yields that there is some $\alpha$ in $\mathfrak{o}_{L}(S)$ such that $\alpha$ is a generator of normal basis of $\mathfrak{o}_{L}(S) / \mathfrak{o}_{k}(S)$ with $\langle\alpha, 1\rangle=1$. If $\mathfrak{b}(\chi)$ is the fractional ideal of $\mathfrak{o}_{k(\chi)}(S)$ depending on $\alpha$ as in (2.1), then we have $\mathfrak{b}(\chi)=(1)$ by [8, Lemma 2.8, (ii)]. Furthermore $p$ is totally ramified in $k_{\chi} / k$ and $k \cap \mathbf{Q}(\chi)=\mathbf{Q}$ by the assumption. So by Proposition 2.3, (ii),

$$
\left(\langle\alpha, \chi\rangle^{g_{x}}\right) \mathfrak{o}_{k(\chi)}(S)=\mathfrak{P}^{\theta},
$$


where $\theta$ is defined in (2.8) and $\mathfrak{P}$ is some prime ideal of $\mathfrak{o}_{k(x)}$ lying above $\mathfrak{p}$. Put $p:=$ $\mathfrak{p} \cap \mathbf{Q}$ and $P:=\mathfrak{B} \cap \mathbf{Q}(\chi)$. Let $b:=b_{\mathfrak{p}}$ and $q$ be the cardinal of $\mathfrak{o}_{\mathbf{Q}(\chi)} / P$ so that $q=p^{b}$ and ${ }^{D_{Q}(x)} / P$ is identified with the field $\mathbf{F}_{q}$ of $q$ elements. Let $\mathrm{T}$ be the trace map from $\mathbf{F}_{q}$ to $\mathbf{F}_{p}$. Define

$$
\psi: \mathbf{F}_{q} \longrightarrow \mathbf{C}^{\times}, \quad \psi(x)=\zeta_{p}^{\mathbf{T}(x)}
$$

Let $\left(\frac{x}{P}\right)_{g_{\chi}}$ be the $g_{\chi}$ th power residue symbol $\bmod P$ in $\mathbf{Q}(\chi)$. Define the Gauss sum

$$
\tau:=-\sum_{x \in \mathbf{F}_{q}^{\times}}\left(\frac{x}{P}\right)_{g_{\chi}}^{-1} \psi(x) .
$$

Let $\Omega:=\operatorname{Gal}(k(\chi) / k)$. Since $p \nmid g_{\chi}$, note that there is a canonical isomorphism:

$$
\Omega \cong \operatorname{Gal}(\mathbf{Q}(\chi) / \mathbf{Q}) \cong \operatorname{Gal}\left(\mathbf{Q}\left(\zeta_{p}\right)(\chi) / \mathbf{Q}\left(\zeta_{p}\right)\right)
$$

By Stickelberger's theorem,

$$
\left(\tau^{g_{\chi}}\right) \mathfrak{o}_{\mathbf{Q}(x)}=P^{\theta} .
$$

Now we establish some relation between Gauss sum $\tau$ and the resolvent $\langle\alpha, \chi\rangle$. As $p \nmid g_{\chi}, p$ is unramified in $\mathbf{Q}(\chi) / \mathbf{Q}$. So $e:=e_{\mathfrak{p}}$ is the ramification index of $\mathfrak{B}$ in $k(\chi) / \mathbf{Q}(\chi)$. Let $Z$ be the decomposition group of $\mathfrak{P}$ in $k(\chi) / \mathbf{Q}(\chi)$ and put $\mathscr{G}:=\operatorname{Gal}(k(\chi) / \mathbf{Q}(\chi))$. Then elements of $\Omega$ and $\mathscr{G}$ are commutative. So by (4.2) and (4.1),

$$
\begin{aligned}
\left(\tau^{g_{x}}\right) \mathfrak{v}_{k(\chi)}(S) & =\left(\prod_{\sigma \in \mathscr{G} / Z} \mathfrak{P}^{\sigma}\right)^{\theta e}=\prod_{\sigma \in \mathscr{G} / Z}\left(\mathfrak{P}^{\theta}\right)^{e \sigma} \\
& =\left(\prod_{\sigma \in \mathscr{G} / Z}\langle\alpha, \chi\rangle^{g_{\chi} e \sigma}\right)_{\mathfrak{v}_{k(\chi)}}(S) .
\end{aligned}
$$

As $g_{\chi}$ is odd, there is some $\omega$ in $\Omega$ such that $\zeta^{\omega}=\zeta^{2}$, where $\zeta$ is a primitive $g_{\chi}$ th root of unity. Let $J:=\tau^{2-\omega}$ (Jacobi sum) in $\mathbf{Q}(\chi)$. Then we have

$$
J \bar{J}=q, \quad J \equiv-1 \bmod (\zeta-1),
$$

where the bar denotes the complex conjugation. Let $A:=\langle\alpha, \chi\rangle^{2-\tilde{\omega}}$ in $k(\chi)$ where $\tilde{\omega}$ is an extension of $\omega$ to $L(\chi)$, and put $B:=\prod_{\sigma \in \mathscr{S} / \mathbf{Z}} A^{\sigma}$. Then since $\langle\alpha, \chi\rangle \equiv\langle\alpha, 1\rangle=$ $1 \bmod (\zeta-1)$, we have $B \equiv 1 \bmod (\zeta-1)$. Furthermore it follows from (4.3) that $(J) \mathfrak{o}_{k(x)}(S)=\left(B^{e}\right) \mathfrak{o}_{k(\chi)}(S)$. Hence there exists some $\varepsilon$ in $\mathfrak{o}_{k(x)}(S)^{\times}$such that

$$
B^{e}=\varepsilon J \text {. }
$$

By the definition of $S$ and Proposition 3.3, the complex conjugation acts trivially on $S$; therefore $\varepsilon \in \mathfrak{o}_{k(\chi)}(S)^{\times}$implies $\bar{\varepsilon} \in \mathfrak{o}_{k(\chi)}(S)^{\times}$and $\operatorname{ord}_{\mathfrak{B}}(\varepsilon / \bar{\varepsilon})=0$ for any prime ideal $\mathfrak{P}$ of $\mathbf{o}_{\boldsymbol{k}(x)}$ lying above $S$. Since $k$ is a totally real number field or a $C M$-field, $k(\chi)$ is a $C M$-field. Hence $\varepsilon / \bar{\varepsilon}$ is a root of unity by the generalized Dirichlet's unit theorem. Let $2^{a} w$ be the 
number of roots of unity in $k(\chi)$ where $w$ is odd. Since $J / \bar{J}=J^{2} / q$, it follows from (4.4) that

$$
(B / \bar{B})^{w e}=\left(J^{w} / q^{w / 2}\right)^{2} \cdot(\varepsilon / \bar{\varepsilon})^{w} .
$$

Since $e$ is even, there is some 2-power root of unity $\xi$ such that

$$
(B / \bar{B})^{w e / 2}= \pm J^{w} /\left(q^{w / 2} \xi\right) \text {. }
$$

It follows from $B \equiv 1, J \equiv-1 \bmod (\zeta-1)$ and $\left(q, g_{\chi}\right)=1$ that

$$
q^{w / 2} \xi \equiv \pm 1 \bmod (\zeta-1)
$$

Let $F:=\mathbf{Q}(\chi)\left(q^{1 / 2}, \xi\right)$. As $b$ is odd, we have $q^{1 / 2} \notin \mathbf{Q}$. Since $\left(2 q, g_{\chi}\right)=1, \operatorname{Gal}\left(\mathbf{Q}\left(q^{1 / 2}, \xi\right) / \mathbf{Q}\right)$ is identified with $\operatorname{Gal}(F / \mathbf{Q}(\chi))$. Therefore by $(2, q)=1$, there is an isomorphism $\varphi$ of $F / \mathbf{Q}(\chi)$ such that $\varphi(\xi)=\xi$ and $\varphi\left(q^{1 / 2}\right)=-q^{1 / 2}$. Applying $\varphi$ to (4.5), since $w$ is odd, we have $1 \equiv-1 \bmod (\zeta-1)$, hence $l=g_{\chi}=2$. This is a contradiction. Thus our theorem is proved.

Proposition 4.3. Assume that $\mathbf{Q} \subset k \subset K \subset \mathbf{Q}\left(\zeta_{p}\right), p$ being an odd prime, and $[k: \mathbf{Q}]$ is even. Suppose that there exists an odd prime $l$ such that $l \mid[K: k]$. Then for any finite subset $S($ or $S=\varnothing)$ of $\mathfrak{\Xi}_{l}, \mathfrak{o}_{K}(S) / \mathfrak{o}_{k}(S)$ does not have a normal basis.

REMARK 4.4. If we assume that $l \equiv 1 \bmod 4$ in the case where $k$ is totally real and [ $k: \mathbf{Q}]$ is a power of 2 , then the set $\mathfrak{S}_{l}$ is always infinite by Lemma 3.5.

Proof of Proposition 4.3. By $(p, l)=1$, we have $k \cap \mathbf{Q}\left(\zeta_{l}\right)=\mathbf{Q}$. Let $\mathfrak{p}$ be the unique prime ideal of $\mathfrak{o}_{k}$ lying above $p$. Since $\mathfrak{p}$ is totally ramified in $K / k$, we have $K \cap \tilde{k}=k$. Furthermore since $\mathfrak{p}$ is tamely ramified and only a prime ideal of $\mathfrak{o}_{k}$ which is ramified in $K / k$, the conductor of $K / k$ is of the form $\mathfrak{p m}_{\infty}$ (therefore $\mathfrak{m}_{2}=1$ ). So $l \mid[K \cap k(\mathfrak{p}): k]$, because $\left[k\left(\mathfrak{p m} m_{\infty}\right): k(\mathfrak{p})\right]$ is a power of 2 by class field theory. Now $e_{\mathfrak{p}}=[k: \mathbf{Q}] \Rightarrow 2 \mid e_{\mathfrak{p}}$ and $p \equiv 1 \bmod l \Rightarrow b_{\mathfrak{p}}=1$; therefore $\operatorname{ord}_{2}\left(b_{\mathfrak{p}}\right)=0$, so that $\mathfrak{p} \in \mathfrak{I}_{l}$. Claim that $\mathfrak{p} \notin S$. This follows from $\mathfrak{I}_{l} \cap \mathfrak{S}_{1, l}=\varnothing$ when $k$ is totally real. When $k$ is a $C M$-field, if $\mathfrak{p} \in S$, then we have $\mathfrak{p} \in \mathfrak{S}_{22, l}\left(\because \mathfrak{I}_{l} \cap \mathfrak{S}_{21, l}=\varnothing\right)$, so that $\mathfrak{p}$ is inert in $k / k^{+}$. This contradicts that $\mathfrak{p}$ is totally ramified in $k / \mathbf{Q}$. Hence $\mathfrak{o}_{K}(S) / \mathfrak{o}_{k}(S)$ does not have a normal basis by Theorem 4.1.

Proposition 4.5. Let $k$ be a quadratic field such that $[\tilde{k}: k]$ is a power of 2 and $\mathfrak{p}$ a prime ideal of $\mathfrak{o}_{k}$ which is ramified in $k / \mathbf{Q}$. Put $p:=\mathfrak{p} \cap \mathbf{Z}$. Suppose that there exists an odd prime $l$ such that $l \mid\left((p-1) / w_{\mathfrak{p}}\right)$, where $w_{\mathfrak{p}}$ is defined in Remark 4.2. Then for any finite subset $S($ or $S=\varnothing)$ of $\mathfrak{S}_{l}, \mathfrak{o}_{k(\mathfrak{p})}(S) / \mathfrak{o}_{k}(S)$ does not have a normal basis.

REMARK 4.6. By Lemma 3.5, the set $\varsigma_{l}$ is always infinite, if we assume that $l \equiv 1 \bmod 4$ and $l$ is prime to the discriminant of $k / \mathbf{Q}$ when $k$ is a real quadratic field.

Proof of Proposition 4.5. Now $e_{\mathfrak{p}}=2, \mathrm{~Np}=p$ and $b_{p}=1$ hold. Since $p \neq l, k \cap$ $\mathbf{Q}\left(\zeta_{l}\right)=\mathbf{Q}$. And we have $\mathfrak{p} \notin S$ by the same reason as in the proof of Proposition 4.3. 
Hence Theorem 4.1 implies our assertion.

\section{Normal integral bases in abelian fields with prime conductors.}

Let $p$ be an odd prime. In this section, we let $K$ be a subfield of the $p$ th cyclotomic field $\mathbf{Q}\left(\zeta_{p}\right)$, and $k$ a subfield of $K$. Let $n:=[K: k](>1)$ and $m:=[k: \mathbf{Q}]$. If $m=1$, then it is well known that $\mathbf{o}_{K} / \mathbf{o}_{k}$ has a normal basis. So we assume that $m>1$ throughout this section. Our goal is Theorem 5.3.

Let $\Gamma:=\operatorname{Gal}(K / \mathbf{Q})$. Since $\Gamma$ is cyclic, so is the group $\hat{\Gamma}$ of its characters; let $\psi_{0}$ be a fixed generator of $\hat{\Gamma}$. There exists a natural surjective group homomorphism:

$$
\hat{\Gamma} \longrightarrow \hat{G},\left.\quad \psi \longmapsto \psi\right|_{G} \cdot
$$

For a positive integer $i$, we put $\psi_{i}:=\psi_{0}^{i}$ and $\chi_{i}:=\left.\psi_{i}\right|_{G}$. Let $l_{i}:=(i / d, m)$ where $d=d_{i}$ is the greatest common divisor of $i$ and $n$. Then

$$
g_{\psi_{i}}=\frac{m}{l_{i}} g_{\chi_{i}},
$$

where $g_{\psi_{i}}\left(\right.$ resp. $g_{\chi_{i}}$ ) is the order of $\psi_{i}$ (resp. $\left.\chi\right)$ in $\hat{\Gamma}$ (resp. $\left.\hat{G}\right)$. For a number field $N$ and each $\psi \in \hat{\Gamma}, N(\psi)$ denotes the field generated by the value of $\psi$ on $\Gamma$ over $N$. Let $\Omega_{i}:=\operatorname{Gal}\left(k\left(\psi_{i}\right) / k\right)$ and $\xi_{i}$ be a fixed primitive $g_{\psi_{i}}$ th root of unity. Since $k \cap \mathbf{Q}\left(\psi_{i}\right)=\mathbf{Q}$ by $\left(p, g_{\psi_{i}}\right)=1$, there exists a group isomorphism $\imath_{i}$ of $\Omega_{i}$ into $\left(Z / g_{\psi_{i}} \mathbf{Z}\right)^{\times}$such that $\xi_{i}^{\omega}=\xi_{i}^{l_{i}(\omega)}$ for all $\omega \in \Omega_{i}$. For each $\omega \in \Omega_{\psi_{i}}$, let $t_{i}(\omega)$ be the integer satisfying $l_{i}(\omega)=t_{i}(\omega) \bmod g_{\psi_{i}}$, $0<t_{i}(\omega)<g_{\psi_{i}}$ and put

$$
\eta_{i}:=\sum_{\omega \in \Omega_{i}}\left[l_{i} t_{i}(\omega) / g_{\chi_{i}}\right] \omega^{-1},
$$

where $[x]$ denotes the greatest integer $\leq x$ as usual for a real number $x$. For each $\psi \in \hat{\Gamma}$, we define the group homomorphism $\operatorname{det}_{\psi}$ by

$$
\operatorname{det}_{\psi}: k \Gamma^{\times} \longrightarrow k(\psi)^{\times}, \quad \sum_{s \in \Gamma} a_{s} s \longmapsto \sum_{s \in \Gamma} \psi(s) a_{s} .
$$

Proposition 5.1. Let $\beta \in \mathfrak{o}_{K}$ be a free generator of $K$ over $k G$. Then there exists some $\lambda$ in $k \Gamma^{\times}$such that for any positive integer $i$ with $i \not \equiv 0 \bmod n$, we have

$$
\mathfrak{b}\left(\chi_{i}\right)^{-1}=\left(\operatorname{det}_{\psi_{i}}(\lambda)\right) \mathfrak{P}_{i}^{\eta_{i}},
$$

where $\mathfrak{P}_{i}$ is some prime ideal of $\mathfrak{o}_{k\left(\psi_{i}\right)}$ lying above $p$ and, taking $S=\varnothing, \mathfrak{b}\left(\chi_{i}\right)$ is the fractional ideal of $\mathfrak{o}_{k\left(\chi_{i}\right)}$ depending on $\beta$ as in (2.1).

ProOF. Let $\alpha:=\operatorname{Tr}_{\mathbf{Q}\left(\zeta_{p}\right) / K}\left(\zeta_{p}\right)$. Since $\alpha$ is a free generator of $K$ over $\mathbf{Q} \Gamma$, we can prove the following in the same way as in Fröhlich [7, Lemma 6.2 and Theorem 25, (ii) of Chapter III]: there exists some $\lambda$ in $k \Gamma^{\times}$such that 


$$
\left\langle\beta,\left.\psi\right|_{G}\right\rangle_{K / k}=\operatorname{det}_{\psi}(\lambda)\langle\alpha, \psi\rangle_{k / \mathbf{Q}},
$$

for all $\psi \in \hat{\Gamma}$. Let $\tilde{\psi}_{i}$ be the character of $\operatorname{Gal}\left(\mathbf{Q}\left(\zeta_{p}\right) / \mathbf{Q}\right)$ of order $g_{\psi_{i}}$, defined by $\tilde{\psi}_{i}(s):=$ $\psi_{i}\left(\left.s\right|_{K}\right)$ for all $s \in \operatorname{Gal}\left(\mathbf{Q}\left(\zeta_{p}\right) / \mathbf{Q}\right)$. Then it follows from the definition of $\alpha$ that

$$
\left\langle\alpha, \psi_{i}\right\rangle_{K / \mathbf{Q}}=\sum_{s \in \operatorname{Gal}\left(\mathbf{Q}\left(\zeta_{p}\right) / \mathbf{Q}\right)} \tilde{\psi}_{i}\left(s^{-1}\right) \zeta_{p}^{s} .
$$

Let $P$ be any prime ideal of $\mathfrak{o}_{\mathbf{Q}\left(\psi_{i}\right)}$ lying above $p$. Since $p \equiv 1 \bmod g_{\psi_{i}}, p$ is completely decomposed in $\mathbf{Q}\left(\psi_{i}\right) / \mathbf{Q}$ so that $\mathfrak{D}_{\mathbf{Q}\left(\psi_{i}\right)} / \boldsymbol{P}$ is identified with the field $\mathbf{F}_{p}$ of $p$ elements. Since $i \neq \equiv \bmod n, g_{\chi_{i}}>1$ so that $g_{\psi_{i}}>1$. Let $\left(\frac{x}{P}\right)_{g_{\psi_{i}}}$ be the $g_{\psi_{i}}$ th power residue symbol $\bmod P$ in $\mathbf{Q}\left(\psi_{i}\right)$ which can be regarded as a character of $\mathbf{F}_{p}^{\times}$of order $g_{\psi_{i}}$. Since $\operatorname{Gal}\left(\mathbf{Q}\left(\zeta_{p}\right) /\right.$ $\mathbf{Q})$ is identified with $\mathbf{F}_{p}^{\times}, \tilde{\psi}_{i}$ is also a character of $\mathbf{F}_{p}^{\times}$of order $g_{\psi_{i}}$. Consequently there is some $\delta$ in $\Omega_{i} \cong\left(\mathbf{Z} / g_{\psi_{i}} \mathbf{Z}\right)^{\times}$such that $\tilde{\psi}_{i}=\left(\frac{}{P}\right)_{g_{\psi_{i}}}^{\delta}$. Define the Gauss sum

$$
\tau:=-\sum_{x \in \mathbf{F}_{p}^{\times}}\left(\frac{x}{P}\right)_{g_{\psi_{i}}}^{-1} \zeta_{p}^{x} .
$$

$\operatorname{As}\left(p, g_{\psi_{i}}\right)=1, \Omega_{i}$ can be identified with $\operatorname{Gal}\left(\mathbf{Q}\left(\zeta_{p}\right)\left(\psi_{i}\right) / \mathbf{Q}\left(\zeta_{p}\right)\right)$. Hence we have $\left\langle\alpha, \psi_{i}\right\rangle_{K / \mathbf{Q}}=$ $-\tau^{\delta}$. Since $P$ is totally ramified in $k\left(\psi_{i}\right) / \mathbf{Q}\left(\psi_{i}\right), P=\mathfrak{P}^{m}$ with some prime ideal $\mathfrak{P}$ of $\mathfrak{o}_{k\left(\psi_{i}\right)}$. Let $\mathfrak{P}_{i}:=\mathfrak{P}^{\delta}$. Then we have by Stickelberger's theorem

$$
\left(\left\langle\alpha, \psi_{i}\right\rangle_{\mathbf{K} / \mathbf{Q}}^{g_{\psi_{i}}}\right)=\mathfrak{P}_{i}^{m \theta_{i}},
$$

where we put $\theta_{i}:=\sum_{\omega \in \Omega_{i}} t_{i}(\omega) \omega^{-1}$. Hence it follows from (5.3) that

$$
\left(\left\langle\beta, \chi_{i}\right\rangle_{K / k}^{g \psi_{i}}\right)=\left(\operatorname{det}_{\psi_{i}}(\lambda)^{g \psi_{i}}\right) \mathfrak{P}_{i}^{m \theta_{i}}
$$

Let $\mathfrak{p}$ be the unique prime ideal of $\mathfrak{o}_{k}$ lying above $p$. Since $\mathfrak{p} \nmid g_{\chi_{i}}$, we have by (2.3) and Proposition 2.3, (i),

$$
\left(\left\langle\beta, \chi_{i}\right\rangle_{\mathbf{K} / \mathbf{k}}^{g_{\chi_{i}}}\right)=\mathfrak{a}\left(\chi_{i}\right) \mathfrak{b}\left(\chi_{i}\right)^{-g_{\chi_{i}}}
$$

and $\mathfrak{a}\left(\chi_{i}\right)$ is a $g_{\chi_{i}}$-power free ideal of $\mathfrak{o}_{k\left(\chi_{i}\right)}$. Hence (5.2) follows from (5.1), (5.4), (5.5) and the definition of $\eta_{i}$. This proves our proposition.

Proposition 5.2. Let $i$ be a positive integer with $i \neq \equiv \bmod n$ and $\beta, \mathfrak{b}\left(\chi_{i}\right)$ as in Proposition 5.1. Under the above notations, assume that $\left(l_{i}, g_{\psi_{i}}\right)=1, l_{i}>1$ and one of the following conditions is satisfied:

(i) $l_{i}$ is odd and $g_{\chi_{i}}>2$,

(ii) $l_{i}$ is even, $l_{i} \geq 4$ and " $l_{i} \neq 6$ or $g_{\chi_{i}} \neq 5$ ".

Then $\mathfrak{b}\left(\chi_{i}\right)$ is not a principal ideal of $\mathfrak{o}_{k\left(\chi_{i}\right)}$.

Proof. Since $l_{i} \mid m$, there exists the unique subfield $F$ of $k$ with $[k: F]=l_{i}$. Let $\mathscr{G}:=\operatorname{Gal}\left(k\left(\psi_{i}\right) / F\left(\psi_{i}\right)\right)$ and $\mathfrak{P}_{i}$ be as in Proposition 5.1. Assume that $\mathfrak{b}\left(\chi_{i}\right)$ is a principal 
ideal of $\mathfrak{o}_{k\left(\chi_{i}\right)}$. So by Proposition 5.1, there is some $A$ in $k\left(\psi_{i}\right)^{\times}$such that $\mathfrak{P}_{i}^{\eta_{i}}=(A)$. Let $\omega_{0} \in \Omega_{i}$ such that $\xi_{i}^{\omega_{0}}=\xi_{i}^{-1}$. Since $\mathfrak{P}_{i}$ is totally ramified in $k\left(\psi_{i}\right) / \mathbf{Q}\left(\psi_{i}\right)$, we have $\overline{\mathfrak{P}_{i}}=\mathfrak{P}_{i}^{\omega_{0}}$ so that $\overline{\mathfrak{P}_{i}^{\eta_{i}}}=\mathfrak{P}_{i}^{\eta_{i} \omega_{0}}$, since $k\left(\psi_{i}\right) / \mathbf{Q}$ is abelian, where the bar denotes the complex conjugation. It is easy to see that $\eta_{i}-\eta_{i} \omega_{0}=\sum_{\omega \in \Omega_{i}}\left\{2\left[l_{i} t_{i}(\omega) / g_{\chi_{i}}\right]+1-m\right\} \omega^{-1}$. Hence we have

$$
\operatorname{ord}_{\mathfrak{P}_{i}}(A / \bar{A})=2\left[l_{i} / g_{\chi_{i}}\right]+1-m .
$$

For a Dedekind domain $\mathfrak{o}$, we denote by $P(\mathfrak{o})$ the group of principal ideals of $\mathfrak{v}$. The group $P\left(\mathfrak{o}_{F\left(\psi_{i}\right)}\right)$ can be regarded as a subgroup of $P\left(\mathfrak{o}_{k\left(\psi_{i}\right)}\right)$ by the extension of ideals. Then $P\left(\mathfrak{o}_{k\left(\psi_{i}\right)}\right)^{\mathscr{G}} / P\left(\mathfrak{o}_{F\left(\psi_{i}\right)}\right)$ is isomorphic to the cohomology group $\mathrm{H}^{1}\left(\mathscr{G}, \mathfrak{o}_{k\left(\psi_{i}\right)}^{\times}\right)$, where $P\left(\mathrm{D}_{k\left(\psi_{i}\right)}\right)^{\mathscr{G}}$ denotes the group of elements of $P\left(\mathrm{o}_{k\left(\psi_{i}\right)}\right)$, fixed by $\mathscr{G}$. Furthermore since $\mathscr{G}$ is cyclic, this cohomology group is isomorphic to ${ }_{N}\left(\mathfrak{o}_{k\left(\psi_{i}\right)}^{\times}\right) /\left(\mathfrak{o}_{k\left(\psi_{i}\right)}^{\times}\right)^{\sigma-1}$, where $\sigma$ is a generator of $\mathscr{G}, \mathbf{N}_{\mathbf{N}}\left(\mathrm{o}_{k\left(\psi_{i}\right)}^{\times}\right):=\left\{u \in \mathfrak{v}_{k\left(\psi_{i}\right)}^{\times} \mid \mathrm{N}(u)=1\right\}$ and $\mathrm{N}$ is the norm map from $k\left(\psi_{i}\right)$ to $F\left(\psi_{i}\right)$. Let $(x) \in P\left(\mathfrak{o}_{k\left(\psi_{i}\right)}\right)$. Then under this group isomorphism, the class of $(x)$ corresponds to the class of $x^{\sigma-1}$, and the class of $(x / \bar{x})$ corresponds to the class of $x^{\sigma-1} / \overline{x^{\sigma-1}}$, since $k\left(\psi_{i}\right)$ is a $C M$-field.

Since $\mathfrak{P}_{i}$ is totally ramified in $k\left(\psi_{i}\right) / F\left(\psi_{i}\right)$ and $k\left(\psi_{i}\right) / F$ is abelian, $\mathfrak{P}_{i}^{\eta_{i}}$ is now fixed by $\mathscr{G}$. So $(A) \in P\left(\mathrm{o}_{k\left(\psi_{i}\right)}\right)^{\mathscr{G}}$. We claim that $(A / \bar{A})$ belongs to $P\left(\mathrm{o}_{F\left(\psi_{i}\right)}\right)$ if $l_{i}$ is odd, and to $\left\langle(\sqrt{a}) \bmod P\left(\mathrm{o}_{F\left(\psi_{i}\right)}\right)\right\rangle$ if $l_{i}$ is even, where $\sqrt{a}\left(a \in F\left(\psi_{i}\right)^{\times}\right)$is a primitive element of the quadratic subextension of $k\left(\psi_{i}\right) / F\left(\psi_{i}\right)$. Put indeed $u:=A^{\sigma-1}$. Since $k\left(\psi_{i}\right)$ is a $C M$-field, $u / \bar{u}$ is a root of unity by Dirichlet's unit theorem. As $k \subsetneq \mathbf{Q}\left(\zeta_{p}\right)$, the group of roots of unity in $k\left(\psi_{i}\right)$ is generated by $\pm \xi_{i}$. So $u / \bar{u}=\left(-\xi_{i}\right)^{v}$ with some integer $v$. Taking the norm $\mathrm{N}$, we see $1=\left(-\xi_{i}\right)^{v l_{i}}$, therefore $2 g_{\psi_{i}} \mid v l_{i}$. Since $\left(l_{i}, g_{\psi_{i}}\right)=1$, we have $2 g_{\psi_{i}} \mid v$ (resp. $g_{\psi_{i}} \mid v$ ), hence $u / \bar{u}=1$ (resp. \pm 1 ) when $l_{i}$ is odd (resp. even). Thus our claim is proved since $\sqrt{a}^{\sigma-1}=-1$. Hence there are some $\varepsilon$ in $\mathfrak{o}_{k\left(\psi_{i}\right)}^{\times}$and some $b$ in $F\left(\psi_{i}\right)^{\times}$such that $A / \bar{A}=\sqrt{a^{j}} b \varepsilon$, where $j=0$ or 1 , and if $l_{i}$ is odd, then we put $j=0$. So

$$
\operatorname{ord}_{\mathfrak{P}_{i}}(A / \bar{A}) \equiv j \frac{l_{i}}{2} \operatorname{ord}_{P_{i}}(a) \bmod l_{i}
$$

where let $P_{i}:=\mathfrak{P}_{i} \cap F\left(\psi_{i}\right)$. It follows from (5.6) that

$$
2\left[l_{i} / g_{\chi_{i}}\right]+1 \equiv j \frac{l_{i}}{2} \operatorname{ord}_{P_{i}}(a) \bmod l_{i}
$$

(i) The case where $l_{i}$ is odd. As $g_{\chi_{i}}>2,2\left[l_{i} / g_{\chi_{i}}\right]+1 \leq 2\left(l_{i}-1\right) / 2+1=l_{i}$. So it follows from $j=0$ and (5.7) that $2\left[l_{i} / g_{\chi_{i}}\right]+1=l_{i}$. Since $\left(l_{i}, g_{\chi_{i}}\right)=1$, we can write $l_{i}=g_{\chi_{i}} q+r$ with some non-negative integer $q$ and $0<r<g_{x_{i}}$. Therefore $\left(2-g_{x_{i}}\right) q=r-1$, so $q=0$, $r=1$. Hence we have $l_{i}=1$. This is a contradiction.

(ii) The case where $l_{i}$ is even. Then it follows from (5.7) that $j(=1), l_{i} / 2$ and $\operatorname{ord}_{P_{i}}(a)$ are all odd. So we have $2\left[l_{i} / g_{\chi_{i}}\right]+1 \equiv l_{i} / 2 \bmod l_{i}$. Since $\left(l_{i}, g_{\chi_{i}}\right)=1$ and $g_{\chi_{i}}>1$, we have $g_{\chi_{i}}>2$, hence $2\left[l_{i} / g_{\chi_{i}}\right]+1=l_{i} / 2$. We write $l_{i}=g_{x_{i}} q+r$ with some non-negative integer $q$ and $0<r<g_{\chi_{i}}$. Then 


$$
\left(4-g_{\chi_{i}}\right) q=r-2 \text {. }
$$

If $r>2$, then $g_{\chi_{i}}<4$ from (5.8). Since $g_{\chi_{i}}$ is odd, $g_{\chi_{i}}=3$ so that $2<r<3$. This is a contradiction. Therefore $r=1$ or 2 . If $r=2$, then $q=0$ by (5.8) so that $l_{i}=2$. This contradicts $l_{i} \geq 4$. If $r=1$, then $g_{\chi_{i}}=5$ and $l_{i}=6$ from (5.8). This is a contradiction. Thus our proposition is proved.

THEOREM 5.3. Under the above notations, we have the following:

(I) $\mathfrak{o}_{K} / \mathfrak{o}_{k}$ does not have a normal basis, except for the following four cases:

(i ) $m$ is even and not a power of 2 , and $n=2$.

(ii) $m$ and $n$ are both powers of 2 .

(iii) $m$ is a power of $q$ and $n$ is a power of $q$ or $2 \times(a$ power of $q)$, with some odd prime $q$.

(iv) $m$ is odd and $n=2$.

(II) In the case (I-iv), $\mathfrak{v}_{K} / \mathfrak{o}_{k}$ has a normal basis. (For the other cases, see the remark below.)

ProOF. Let $\beta \in \mathfrak{o}_{K}$ be a free generator of $\mathfrak{o}_{k_{\mathfrak{p}}} \bigotimes_{\mathfrak{o}_{k}} \mathfrak{o}_{k}$ over $\mathfrak{o}_{k_{\mathrm{p}}} G$ for each prime ideal $\mathfrak{p}$ of $\mathfrak{o}_{k}$, dividing the order of $G$.

(I) By Proposition 4.3, we need prove when (A): $m$ is even and $n$ is a power of 2, or (B): $m$ is odd.

The case (A). Let $v:=\operatorname{ord}_{2}(m)$ and $i:=m / 2^{v}$. Then $l_{i}=i, g_{\chi_{i}}=n /(i, n)=n$ so that $\left(l_{i}, g_{\psi_{i}}\right)=1$ by (5.1). Since we make exceptions of the cases (ii) and (i), we have $l_{i}>1$ so that $g_{\chi_{i}}>2$. Therefore it follows from Proposition 5.2, (i) that $\mathfrak{b}\left(\chi_{i}\right)$ is not a principal ideal of $\mathfrak{o}_{k\left(\chi_{i}\right)}$. Hence $\mathfrak{o}_{K} / \mathfrak{o}_{k}$ does not have a normal basis by [8, Theorem 2.10, (ii)].

The case (B). If $n$ is not a power of 2 , then there is some odd prime $q$ with $q \mid n$. Let $v:=\operatorname{ord}_{q}(m)(\geq 0)$. When $m / q^{v}>1$, putting $i:=m n / q^{v+1}$, we have $l_{i}=m / q^{v}>1$, $g_{\chi_{i}}=q>2,\left(l_{i}, g_{\psi_{i}}\right)=1$ so that $\mathfrak{b}\left(\chi_{i}\right)$ is not principal by Proposition 5.2, (i). When $m=q^{v}$, let $w:=\operatorname{ord}_{q}(n)$ and $i:=q^{v+w}$. Then $l_{i}=m>1, g_{\chi_{i}}=n / q^{w},\left(l_{i}, g_{\psi_{i}}\right)=1$. Since we make exception of the case (iii), $n / q^{w}>2$ so that $g_{\chi_{i}}>2$. Hence $\mathfrak{b}\left(\chi_{i}\right)$ is not principal by Proposition 5.2, (i). If $n$ is a power of 2 , then we put $i:=m$. So $l_{i}=m>1, g_{\chi_{i}}=n$, $\left(l_{i}, g_{\psi_{i}}\right)=1$. Since we make exception of the case (iv), $n>2$ so that $g_{\chi_{i}}>2$. Hence $\mathfrak{b}\left(\chi_{i}\right)$ is not principal by Proposition 5.2, (i). Thus $\mathfrak{o}_{K} / \mathfrak{o}_{k}$ does not have a normal basis by $[8$, Theorem 2.10, (ii)].

(II) Let $i:=m$. Then $g_{\chi_{i}}=g_{\psi_{i}}=2, l_{i}=m, \Omega_{i}=\{1\}$. So $\hat{G}=\left\{1, \chi_{i}\right\}$. Put $\pi:=$ $\mathrm{N}_{\mathbf{Q}\left(\zeta_{p}\right) / k}\left(1-\zeta_{p}\right)$ so that $\mathfrak{P}_{i}=(\pi)$. As $\eta_{i}=(m-1) / 2$, it follows from (5.2) that

$$
\mathfrak{b}\left(\chi_{i}\right)^{-1}=\left(\pi^{(m-1) / 2} \operatorname{det}_{\psi_{i}}(\lambda)\right) .
$$

From (5.3), $\langle\beta, 1\rangle_{K / k}=\operatorname{det}_{1}(\lambda) \operatorname{Tr}_{\mathbf{Q}\left(\zeta_{p}\right) / \mathbf{Q}}\left(\zeta_{p}\right)=-\operatorname{det}_{1}(\lambda)$. Since $\mathfrak{b}(1)^{-1}=\left(\langle\beta, 1\rangle_{K / k}\right)$ by $[8$, Remark 2.12], we have

$$
\mathfrak{b}(1)^{-1}=\left(\operatorname{det}_{1}(\lambda)\right) \text {. }
$$

It follows from the definition of $\beta$ and [8, Lemma 2.8, (ii)] that any prime divisor of 
$\mathfrak{b}(1)$ and $\mathfrak{b}\left(\chi_{i}\right)$ does not divide two. Let $u:=\zeta_{p}+\zeta_{p}^{-1}$ which is a unit in $\mathbf{Q}\left(\zeta_{p}\right)^{+}$. Since $m$ is odd, $k \subset \mathbf{Q}\left(\zeta_{p}\right)^{+}$. As $u \equiv \mathbf{N}_{\mathbf{Q}\left(\zeta_{p}\right) / \mathbf{Q}\left(\zeta_{p}\right)^{+}}\left(1-\zeta_{p}\right) \bmod 2$, we have $\mathbf{N}_{\mathbf{Q}\left(\zeta_{p}\right)^{+/ k}}(u) \equiv \pi \bmod 2$. Let $\varepsilon:=\mathbf{N}_{\mathbf{Q}\left(\zeta_{p}\right)^{+/ k}}(u)^{-(m-1) / 2} \in \mathfrak{o}_{k}^{\times}$. So we have $\varepsilon \pi^{(m-1) / 2} \equiv 1 \bmod 2$. Since $\operatorname{det}_{1}(\lambda) \equiv \operatorname{det}_{\psi_{i}}(\lambda)$ $\bmod 2$ by $g_{\psi_{i}}=2$,

$$
\operatorname{det}_{1}(\lambda)-\varepsilon \pi^{(m-1) / 2} \operatorname{det}_{\psi_{i}}(\lambda) \equiv 0 \bmod 2 .
$$

Hence by [8, Remark 2.11], $\mathfrak{o}_{K} / \mathfrak{o}_{k}$ has a normal basis. Thus our theorem is proved.

REMARK 5.4. Let $K:=\mathbf{Q}\left(\zeta_{p}\right)$ and $k:=\mathbf{Q}\left(\zeta_{p}\right)^{+}$with $p \equiv 1 \bmod 4$. So $n=2$ and $m$ is even. Then it is well known that $\zeta_{p}$ is a generator of normal basis of $\mathfrak{o}_{K} / \mathfrak{o}_{k}$ (in the cases $(\mathrm{I}-\mathrm{i}, \mathrm{ii}))$. In the case (I-ii), if $n=2$, then we can prove that $\mathfrak{o}_{K} / \mathfrak{o}_{k}$ has a normal basis. In the case (I-iii), $\mathfrak{o}_{K} / \mathfrak{o}_{k}$ does not have a normal basis by Brinkhuis [1, Theorem 4.1], because a sequence of Galois extension $\mathbf{Q} \subset k \subset K$ does not split and [k:Q] is odd. The question is still open as to other cases.

Let $S$ be any finite set of prime ideals of $\mathfrak{o}_{k}$ which contains the unique prime ideal of $\mathfrak{o}_{k}$ lying above $p$ and assume that $(m, n)=1$. Then it is easy to see that $\mathfrak{o}_{K}(S) / \mathfrak{o}_{k}(S)$ has a normal basis.

\section{References}

[ 1 ] J. BRINKhUIS, Normal integral bases and embedding problems, Math. Ann. 264 (1983), 537-543.

[2] - Normal integral bases and complex conjugation, J. Reine Angew. Math. 375(1987), 157-166.

[ 3] J. Cassels and A. Fröhlich (ed.), Algebraic Number Theory, Academic Press (1967).

[4] J. Cougnard, Quelques extensions modérément ramifiées sans base normale, J. London Math. Soc. 31 (1985), 200-204.

[ 5 ] - Bases normales relatives dans certaines extensions cyclotomiques, J. Number Theory 23 (1986), 336-346.

[6] A. FröHLICH, Stickelberger without Gauss sums, Algebraic Number Fields (Proceedings of The Durham Symposium 1975), Academic Press (1977), 589-607.

[7] - Galois Module Structure of Algebraic Integers, Springer (1983).

[ 8] F. KaWamoto and K. Komatsu, Normal bases and $\mathbf{Z}_{p}$-extensions, J. Algebra 163 (1994), $335-347$.

[9] B. SodAÏGUI, Structure galoisienne relative des anneaux d'entiers, J. Number Theory 28 (1988), 189-204.

Present Address:

Department of Mathematics, GakUShUin University,

Mejiro, TOSHIMA-KU, TOKYo, 171 JAPAN. 